\title{
Segregation of striated and smooth muscle lineages by a Notch-dependent regulatory network
}

\author{
Mordechai Applebaum¹, Raz Ben-Yair ${ }^{1,2}$ and Chaya Kalcheim ${ }^{1 *}$
}

\begin{abstract}
Background: Lineage segregation from multipotent epithelia is a central theme in development and in adult stem cell plasticity. Previously, we demonstrated that striated and smooth muscle cells share a common progenitor within their epithelium of origin, the lateral domain of the somite-derived dermomyotome. However, what controls the segregation of these muscle subtypes remains unknown. We use this in vivo bifurcation of fates as an experimental model to uncover the underlying mechanisms of lineage diversification from bipotent progenitors.

Results: Using the strength of spatio-temporally controlled gene missexpression in avian embryos, we report that Notch harbors distinct pro-smooth muscle activities depending on the duration of the signal; short periods prevent striated muscle development and extended periods, through Snail1, promote cell emigration from the dermomyotome towards a smooth muscle fate. Furthermore, we define a Muscle Regulatory Network, consisting of Id2, Id3, FoxC2 and Snail1, which acts in concert to promote smooth muscle by antagonizing the pro-myogenic activities of Myf5 and Pax7, which induce striated muscle fate. Notch and BMP closely regulate the network and reciprocally reinforce each other's signal. In turn, components of the network strengthen Notch signaling, while Pax7 silences this signaling. These feedbacks augment the robustness and flexibility of the network regulating muscle subtype segregation.
\end{abstract}

Conclusions: Our results demarcate the details of the Muscle Regulatory Network, underlying the segregation of muscle sublineages from the lateral dermomyotome, and exhibit how factors within the network promote the smooth muscle at the expense of the striated muscle fate. This network acts as an exemplar demonstrating how lineage segregation occurs within epithelial primordia by integrating inputs from competing factors.

Keywords: Avian embryo, Id, FoxC2, Snail, BMP, Epithelial-to-mesenchymal transition, Myotome, Somite

\section{Background}

The hallmark of multicellular organisms is the division of labor of its constituent cells and tissues. Differentiation of identical progenitors into a range of cell types with striking diversity of functions is a basic theme in development, as in evolution [1]. In this context, understanding the mechanisms underlying emergence of distinct muscle sublineages from multipotent stem cells is of critical interest in developmental research, with growing pertinence to congenital abnormalities and adult pathologies $[2,3]$.

\footnotetext{
* Correspondence: kalcheim@cc.huji.ac.il

${ }^{1}$ Department of Medical Neurobiology, IMRIC and ELSC, Hebrew University of Jerusalem-Hadassah Medical School, Jerusalem 9112102, P.O. Box 12272, Israel Full list of author information is available at the end of the article
}

Most body muscles develop from the paraxial mesoderm through intermediate pseudostratified epithelial structures called somites. The ventral aspect of somites dissociates first to generate the sclerotome, the precursor of cartilage and bones along the body axis. The dorsal aspect, termed dermomyotome (DM), remains transiently epithelial and contributes primarily to cells in the myotome (future striated muscles and satellite cells) and dermis until completely dissociated $[4,5]$.

The lateral DM is a particularly significant domain as it is the source of appendicular muscles at the fore- and hind-limb levels [6,7] as well as contributing endothelial and smooth muscle (SM) cells in the limbs [8-10]. At non-limb levels the lateral DM contributes to hypaxial skeletal muscles (abdominal and intercostal) [11-13] and 
is also a source of endothelial and SM cells in adjacent blood vessels [2]. Moreover, single-cell lineage analysis in this domain revealed that myotomal and SM cells share a common progenitor within the epithelial primordium [14], therefore providing an attractive model to investigate the mechanisms of fate segregation.

Extrinsic signals such as bone morphogenetic protein (BMP) from the lateral plate mesoderm (LPM) and local cues such as Notch act upon cells of the lateral DM to determine which differentiation program to initiate $[14,15]$. Notch was shown to stimulate cell migration from the lateral DM towards blood vessels where it promoted SM differentiation, whereas cells in which Notch signaling was silenced translocated into the myotome and initiated myogenesis [14]. BMP4 from the LPM was shown to delay the myogenic program by inhibiting the onset of $M y o D$ transcription in progenitors that translocated from the lateral DM into the myotome [11]. However, the downstream mechanisms by which lateral DM progenitors integrate and interpret these signaling cues are unknown.

Id2 and Id3 are basic helix-loop-helix (bHLH) molecules that lack the basic domain and dimerize with bona fide bHLH transcription factors (TF) to repress their activity [16]. As the four myogenic regulatory factors (MRF) Myf5, MyoD, myogenin and MRF4 - are bHLH TFs they are putative targets for Id inhibition. Indeed, Id proteins bind MyoD and inhibit activation of its target genes [16]. Furthermore, $I d$ genes are direct targets of BMP in embryonic stem cells [17] although the activity and regulation of the Id genes in somites is largely unknown [18].

FoxC2 is a member of the Forkhead box TF family and is involved in epithelial-to-mesenchymal transition (EMT) of metastatic cells $[19,20]$. It is expressed in the somite $[21,22]$ and has been implicated in Notch signaling [23-25]. Mutual repression with Pax3/7 has been shown to determine myogenic (high Pax3/7:FoxC2 ratio) or vascular (low Pax3/7:Foxc2 ratio) fates in the murine DM [22].

Snail1 is a Zn-finger TF, known to induce EMT [26]. It is expressed in paraxial mesoderm [27] and promotes dissociation of the central DM [28], but whether it functions in the lateral DM has not been determined.

In this study, we describe a minimal Muscle Regulatory Network (MRN) whereby $I d 2, I d 3, F o x C 2$ and Snail1 are both necessary and sufficient for promoting $\mathrm{SM}$ at the expense of myotomal fates. In addition, Id2/Id3 silence the myogenic activity of Myf5 and upregulate FoxC2 expression. FoxC2 in turn represses Pax7, altogether inhibiting skeletal myogenesis. This MRN operates downstream of both Notch and BMP signaling which positively reinforce each other's activities. Thus, our data assemble a complex network of factors and interactions that are at the core of lineage segregation in the lateral DM.

\section{Results}

Notch signaling has been implicated in the promotion of SM at the expense of striated muscle development from bipotent progenitors resident in the lateral DM [14]. To further assess whether endogenous Notch is necessary for this choice, its activity was inhibited in the lateral DM by focal electroporation (EP) of a dominantnegative (dn) form of MAML1, a co-factor within the Notch activation complex, or Dll1, which represses Notch signaling cell-autonomously [see Additional file 1: Figure S1A-C] [29-31]. Forty hours post-EP, control GFP-labeled cells were observed in the desmin-positive myotome and in the ventral sclerotome between the lateral DM and the cardinal vein likely en route to this blood vessel [see Additional file 1: Figure S1D and [14]]. In addition, a fraction of labeled cells had integrated into the blood vessel wall as SM cells as determined by expression of both desmin and smooth muscle actin (SMA) [see Additional file 1: Figure S1D-D"]. In striking contrast, inhibiting Notch activity promoted an increase in the proportion of myotomal colonization. Consistently, a marked decrease of the proportion of migratory cells was apparent in the sclerotome and blood vessels [see Additional file 1: Figure S1E-G]. These findings are in agreement with previously reported effects of Numb and further confirm a function of Notch in the segregation of muscle sublineages [14,32].

Since SM development requires that progenitors emigrate from their epithelium of origin, migrate through the sclerotome and reach the target blood vessels, we asked whether different phases of this process depend upon the duration of Notch signaling. To this end, we first expressed a constitutively active form of Notch2 (aN2) for increasing periods of time in the lateral DM. As soon as four hours after EP, Notch signaling compromised the epithelial morphology of treated cells, evident by the loss of their typical elongated, pseudostratified appearance, and induced cell delamination when compared to control embryos (Figure 1A,D; $79.6 \pm 2.5 \%$ in control DM compared to $56.7 \pm 7.9 \%$ in Notch-treated embryos; $6 \pm 2.9 \%$ in sclerotome of control embryos compared to $28.2 \pm 6.2 \%$ in Notch-treated embryos, $\mathrm{N}=4$ for each treatment, $P<0.05)$. This was further enhanced by eight hours post-EP (Figure 1B,E; $75.6 \pm 3.3 \%$ in control DM compared to $40.9 \pm 5.1 \%$ in Notch-treated embryos; $13.5 \pm 5.2 \%$ in sclerotome of control embryos compared to $44.8 \pm 6.6 \%$ in Notch-treated embryos, $\mathrm{N}=4$ and 7 , respectively, $P \leq 0.01)$. By 20 hours virtually all labeled cells had delaminated from the DM when compared to controls in which cells were still epithelial (Figure 1C,F; $63.7 \pm 3.2 \%$ in control DM compared to $20.3 \pm 5.7 \%$ in Notch-treated embryos; $16.4 \pm 1.5 \%$ in sclerotome of control embryos compared to $70.5 \pm 5.7 \%$ in Notchtreated embryos, $\mathrm{N}=6$ and 5 , respectively, $P<0.01$ ). 


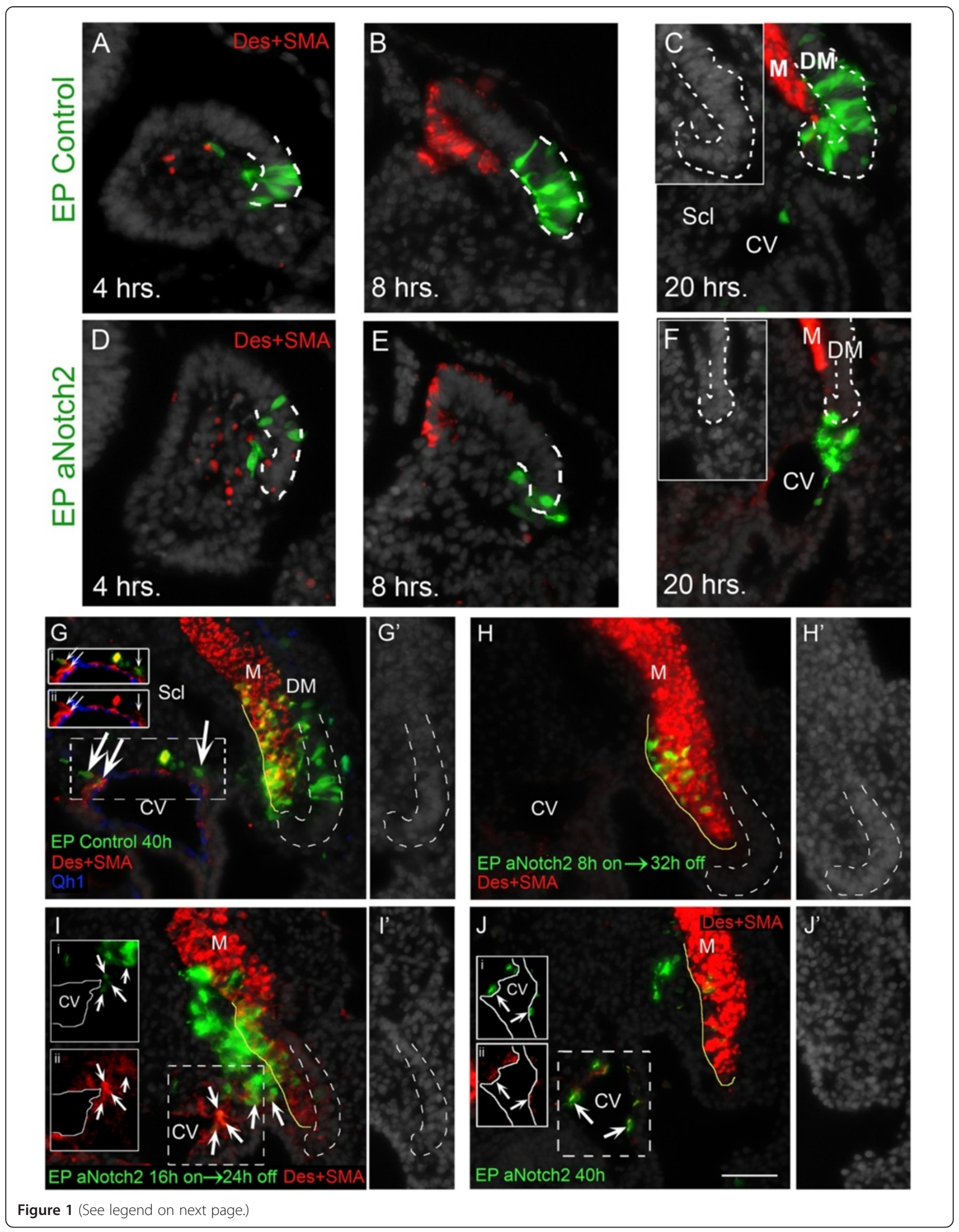


(See figure on previous page.)

Figure 1 Time course of Notch activity in the lateral DM. (A-C) Electroporation of the lateral DM at E2. Control cells are epithelial and located in lateral dermomyotome (DM) both at four hours (A) and eight hours (B) post-electroporation ( $N=4$ and 4, respectively). (C) By 20 hours, although most cells reside in the DM, some have delaminated into an intermediate zone between the lateral DM and myotome ( $\mathrm{N}=6$ ). Inset shows lateral DM by Hoechst staining. (D-F) Electroporation of aNotch2. By four hours and eight hours the epithelial morphology of labeled cells is abrogated and delamination from the lateral DM begun ( $N=4$ and 7, respectively). (F) At 20 hours cells have migrated towards, and partly arrived at, the cardinal vein (CV) ( $=5$ ). Inset shows lateral DM by Hoechst staining. (G-J) Transient Notch activation. (G) Control GFP expressed for 40 hours reveals labeled progeny in myotome (M) and CV (arrows and insets i and ii, $N=8$ ), with residual cells still populating the lateral DM. Qh1 staining of endothelial cells is in blue. $\mathbf{( H )}$ Eight hours of aNotch2 missexpression +32 hours of Tet-off. Cells are clustered in the ventro-medial portion of the myotome, do not express desmin/SMA or display myofiber morphology (see Figure 2A,B) ( $\mathrm{N}=5$ ). (I) Sixteen hours of aNotch $2+24$ hours Tet-off. Most labeled cells are outside the myotome and many of them express ectopic desmin/SMA while others have integrated into the CV wall (arrows and insets i and ii, $\mathrm{N}=5$ ). (J) aNotch2 missexpression for 40 hours. Many Notch + cells co-expressing desmin/ SMA are located in the wall of the CV (arrows and insets i and ii), while others are still on their way through the sclerotome ( $\mathrm{N}=3$ ). Lateral DM in C,F and G-I is marked by a dashed white line. Myotome is outlined with a yellow solid line $\left(\mathbf{G}^{\prime}, \mathbf{H}^{\prime}, \mathbf{I}^{\prime}, \mathbf{J}^{\prime}\right)$ Hoechst staining of lateral DM. Bar: (A-F) 65 Mm; (G-J) $100 \mu \mathrm{m}$. Des, desmin; DM, dermomyotome; SMA, smooth muscle actin.

We next asked whether varying lengths of exposure affect final cell identities. To this end, an inducible version of aNotch2 (aN2) was prepared by subcloning into a tetracycline-sensitive plasmid followed by transfection into the lateral DM. Its expression was restricted by doxycycline treatment to the first 8 hours, 16 hours or 40 hours of a 40-hour total incubation period. Control-GFP-treated embryos exhibited a typical distribution of labeled cells (Figure 1G). Although eight hours exposure of aN2 induced excess delamination from the DM (Figure 1E), it did not prevent myotome colonization (Figure $1 \mathrm{H}$ ). However, these cells were not dispersed throughout the myotome as in controls, but rather aggregated at a ventromedial bulge abutting the sclerotomal border (Figure $1 \mathrm{H}$ ). When exposed to aN2 for 16 hours, most labeled cells were in the sclerotome where they ectopically upregulated SMA/desmin, a feature never observed under control conditions, and some had already incorporated into the wall of the cardinal vein (Figure 1I). To discriminate whether this effect is due to the duration of Notch activity or, alternatively, to a positional effect induced within the sclerotome on cells initially stimulated to emigrate by Notch, Notch was conditionally activated twenty hours post-EP for eight hours only followed by fixation. Under control conditions, the few cells that exited the lateral DM by 28 hours did not express SMA/desmin [see Additional file 2: Figure S2A,A' and [14]]. Late exposure to aN2 stimulated cell delamination from the DM yet was not sufficient to induce the muscle markers in cells located within the ventral sclerotome [see Additional file 2: Figure 2B,B']. Thus, the ectopic muscle marker expression observed (Figure 1I) is likely a consequence of extended Notch activity rather than of an environmental effect stemming from the sclerotome through which the cells migrate.

Continuous expression for 40 hours completely prevented myotome colonization and most cells were already found in the cardinal vein co-expressing SM markers (Figure 1J).
Since a short eight hour exposure to aN2 induced excess delamination from the DM but did not prevent myotome colonization (Figure $1 \mathrm{H}$ ), we sought to further examine the identity of these cells. Whole mount analysis confirmed that these cells translocated into the myotome but additionally showed that they failed to differentiate into unit-length myofibers when compared to their control GFP counterparts (Figure 2A-B). Furthermore, in contrast to control cells in which the progenitor marker Pax7 is downregulated upon exit from the DM (Figure 2C,C',G), eight hours of Notch activity was sufficient to maintain low Pax7 expression for at least sixteen hours post-EP (Figure 2D,D',G). Notch overexpression also maintained low Pax7 expression non-cell autonomously in untransfected cells that exited the DM. This might result from induction of Notch ligand(s) in cells adjacent to the transfected ones, which in turn stimulate Notch activity, that maintains Pax7, in cells neighboring them (Figure 2D,D', arrowhead). In addition, short exposure to Notch stimulated proliferation of lateral DM cells, apparent by enhanced bromodeoxyuridine (BrdU) incorporation (Figure 2E,F,H). Taken together, short and limited exposure to Notch maintains the progenitor state and prevents differentiation. Therefore, Notch signaling harbors two sequential functions. First, with initial signaling it prevents striated myogenesis. Second, with extended signaling, it activates the SM program.

\section{Id2, Id3, FoxC2 and Snail1 affect the choice between striated and SM issued from the lateral DM Transient expression of Id2, Id3, FoxC2 and Snail1 in the lateral DM}

To begin examining how Notch induces SM fate we surveyed for putative genes involved in segregation of muscle sublineages. First, in situ hybridization (ISH) was performed on the flank level of avian embryos to screen for candidate genes expressed in the lateral domain of the DM. Id2, Id3, FoxC2 and Snail1 mRNAs were already 

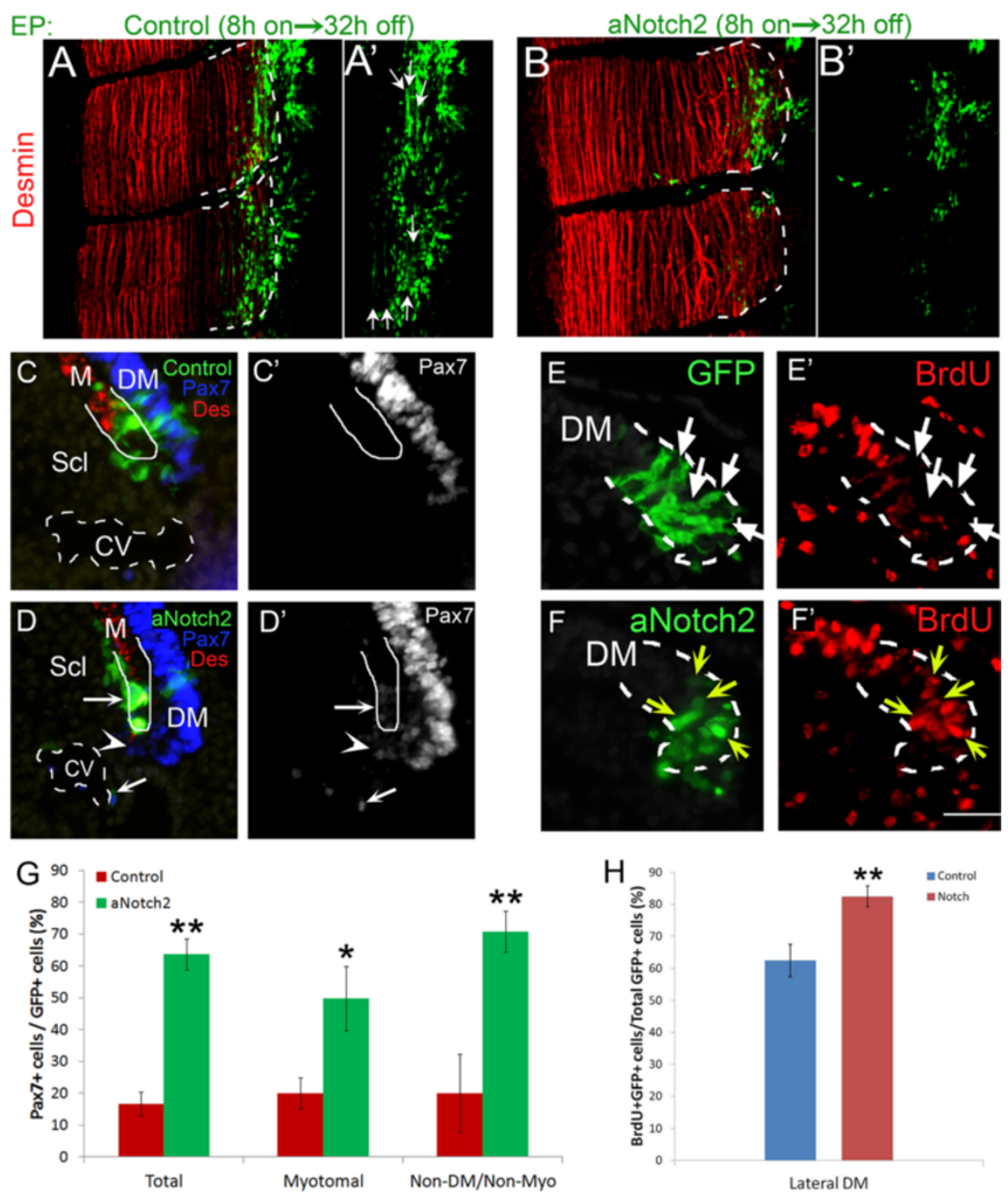

Figure 2 Short exposure to Notch signaling permits myotomal colonization but not myogenic differentiation. $(A, B)$ Dorsal view of whole-mount segments. (A,A') Presumptive lateral DM's were electroporated with an inducible GFP control plasmid. Eight hours post-electroporation doxycyclin was administered to silence plasmid expression. A further 32 hours of incubation proceeded. Cells have initiated myogenesis and begun elongating to form terminally differentiated myofibers $(N=4)$. $\left(\mathbf{B}, \mathbf{B}^{\prime}\right)$ aNotch2 was expressed for 8 hours and an additional 32 hours of incubation without expression followed. Cells entered the desmin + myotome but failed to differentiate into myofibers $(N=4)$. (C-D) Notch maintains Pax7 expression after delamination. (C) Transverse sections of control-GFP-treated embryos 16 hours post-electroporation. Cells that entered the myotome mostly silenced Pax7 expression ( $N$ =4). (D) Transverse sections of aNotch2-treated embryos 16 hours post-electroporation. Cells maintained low Pax7 immunoreactivity even after exiting the DM (arrow). A non-cell autonomous maintenance of Pax7 expression was also observed (arrowhead) $(\mathrm{N}=8)$. ( $(\mathbf{E}-\mathrm{F})$ Notch enhances the incorporation of BrdU into nuclei at the lateral DM. Control-GFP $\left(\mathbf{E}, \mathbf{E}^{\prime}\right)$ or aNotch2 $\left(\mathbf{F}, \mathbf{F}^{\prime}\right)$ was electroporated into the lateral DM and proliferation was monitored eight hours post-treatment. Short exposure to Notch enhances BrdU incorporation in the DM (F, $F^{\prime}$ yellow arrows compared with BrdU-negative nuclei in E, $E^{\prime}$, arrows). (G) Quantification of Pax7-expressing cells (mean \pm SEM). (H) Quantification of BrdU + labeled cells within the lateral DM (mean \pm SEM). ${ }^{*} P \leq 0.05,{ }^{* *} P \leq 0.01$. Bar: (C-F) $50 \mu \mathrm{m}$. BrdU, bromodeoxyuridine; CV, cardinal vein; Des, desmin; DM, dermomyotome; M, myotome; Scl, sclerotome; SEM, standard error of the mean.

apparent at embryonic day (E)2.0 in the lateral aspect of the dorsal epithelial somite that is destined to become the DM [see Additional file 3: Figure S3A-D]. At E2.5 these factors were enriched in the lateral region of the DM, while being also transcribed to different extents in its medial aspect [see Additional file 3: Figure S3E-H]. At
E3.5, although the lateral DM is still epithelial, it has completely repressed their expression [see Additional file 3: Figure S3, arrows in I-L]. Hence, these factors are transcribed early when the lateral DM mostly generates vascular SM fates and are downregulated when this region primarily generates myotomal muscle [14]. This prompted 
the hypothesis that these four factors might be involved in the segregation of vascular and myotomal muscle lineages issued from the lateral DM.

\section{Transient missexpression of Id2, Id3, FoxC2 and Snail1} promotes SM at the expense of myotomal fates issued from the lateral DM

To begin investigating their function(s), we first adopted a gain of function approach. Tetracycline-inducible Id2, Foxc2 or Snail1 were missexpressed in the lateral somite at E2 for 20 hours, a time window corresponding to their endogenous transcription, and re-incubated for an additional day. In control-GFP-treated embryos, the majority of labeled progeny was located in the myotome. Cells were also observed in the ventral sclerotome and a small proportion already integrated into the blood vessel walls as desmin+/SMA + SM right outside the Qh1+ layer of endothelium (Figure 3A,F and Additional file 4: Figure S4A). In contrast, Id2, Foxc2 or Snail1 inhibited cell translocation into the myotome with an increased proportion of transfected cells in the sclerotome and at the SM layer of blood vessels as Desmin+/SMA + cells (Figure 3B,D,E,F and Additional file 4: Figure S4A).

When transiently missexpressed, Id3 had a weaker effect, yet constitutive expression for 44 hours yielded a similar outcome as that obtained with transient activation of Id2, Foxc2 or Snail1, whereby non-myotomal fates, particularly $\mathrm{SM}$, were induced at the expense of the myotomal lineage (Figure 3C,F and Additional file 4: Figure S4A).

Since both Foxc2 and Snail1 also significantly reduced the proportion of progenitors that remained in the DM (Figure 3F), we examined an earlier time point to assess possible effects on cell delamination from the DM epithelium. Already by 16 hours, missexpression of Foxc2 induced EMT of labeled cells from the DM and promoted their migration through the sclerotome [see Additional file 5: Figure S5A,B,E]. An even stronger effect was observed with Snail1, which, in addition to loss of epitheliality, marked by ZO-1 staining, completely prevented myotome colonization [see Additional file 5: Figure S5C-E]. Thus, both Foxc2 and Snail1 trigger EMT of lateral DM progenitors as part of the generation of SM.

\section{Attenuation of Id2/3, FoxC2 and Snail1 activities stimulates myotome formation at the expense of SM}

To examine the physiological functions of the genes, specific inhibitory RNAs were implemented. Inhibitory double-stranded RNAs (dsRNAs) directed against Id2 and Id3 were focally electroporated into the lateral DM. Analysis performed 40 hours post-EP revealed that

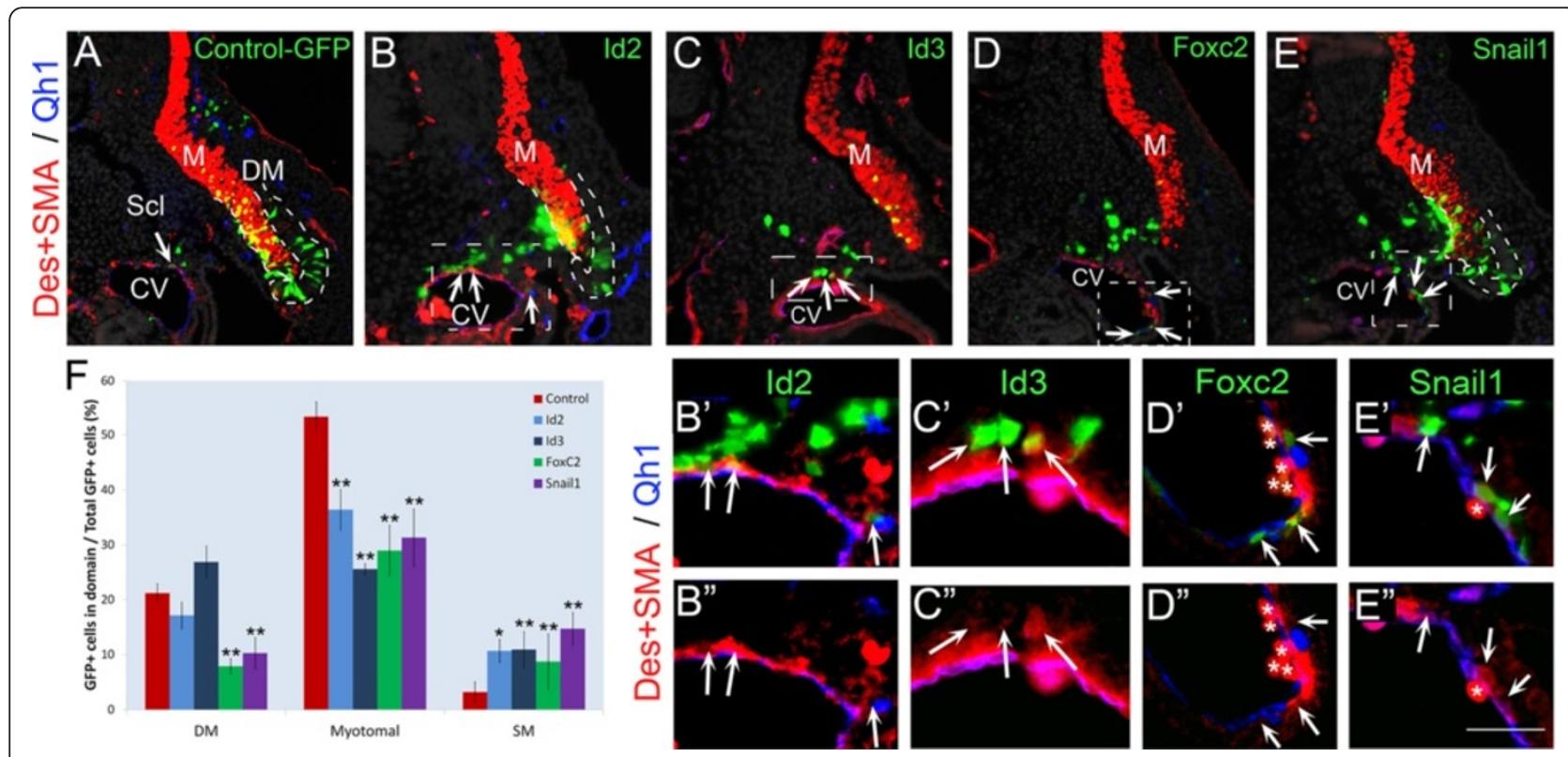

Figure 3 Transient missexpression of Id2, Id3, Foxc2 or Snail1 is sufficient to promote vascular at the expense of myotomal fates. (A) pBI-TRE-EGFP and pRev-Tet-off were co-electroporated into E2 avian somites targeting the presumptive lateral DM. Doxycyclin was administered 20 hours post-treatment and embryos were re-incubated for 24 hours. GFP + cells were found primarily in the myotome (M), co-expressing SMA/ Desmin. A smaller fraction was found in the sclerotome and in blood vessel walls as SM cells (arrow) $(\mathrm{N}=26)$. Transient over-expression of Id2 (B-B", $N=19)$, Foxc2 (D-D", $N=12)$ or Snail1 (E-E", $N=7$ ) or constant expression of Id3 (C-C", $N=3$ ) promoted an increase of cells within the sclerotome as well as SM (arrows and see also Additional file 4: Figure S4A). Endothelial cells were visualized with the Qh1 antibody (blue). (F) Quantifications (mean \pm SEM). Distribution of GFP + cells in various locations as a percentage of total GFP + cells. ${ }^{*} P \leq 0.05$; ${ }^{* *} P \leq 0.01$. Bar: (A-E)

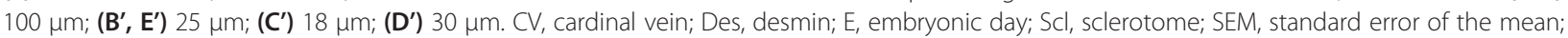
SMA, smooth muscle actin. 
inhibition of Id2/3 preserved a higher proportion of cells in the lateral DM and reduced their exit towards the sclerotome, when compared to the behavior of DMs that received a control dsRNA. Consequently, a lower proportion of labeled cells reached the SM layer of blood vessels (Figure 4A,B,D and Additional file 4: S4B). In addition, dsRNAs to Id2/3 stimulated premature myocyte differentiation that was already apparent 16 hours after transfection [see Additional file 6: Figure S6A,B]. Both short and longterm effects were completely rescued by co-transfecting the dsRNAs with exogenous Id2/3 [see Additional file 6: Figure S6C-E].

Repressing FoxC2 activity with a dsRNA or with a dominant negative FOXC2-Engrailed fusion protein resulted in an increased proportion of GFP-labeled, desmin + myotomal cells out of total labeled cells, at the expense of SM (Figure 4A,C,D and Additional files 4 and 7: S4B, S7A-E). Moreover, precocious myotome colonization and presence of partial as well as full-length myocytes was already observed 16 hours after EP when virtually no myofibers were yet observed in controls [see Additional file 7 : Figure S7A,B,F,G]. Introducing exogenous Foxc2 completely abrogated the effects of Foxc2 knock-down by the dsRNA on myotome colonization [see Additional file 7: Figure S7H-I compared to Figure 4A,C]. Interestingly, both over-expression and knock-down of FoxC2 strongly reduced the proportion of cells remaining in DM (Figures 3D,F; 4C,D and Additional file 7: S7), but while the former promoted the SM fate, the latter induced the myotomal fate, consistent with previous mouse data [22].

Similarly, repressing Snail1 activity with siRNAs designed against Snail1 inhibited cell migration through the sclerotome and subsequent SM development (Figure 4E-G and Additional file 7: Figure S4C). As expected, loss of Snail1 maintained cells within the lateral epithelium (Figure 4E-G, Additional file 8: S8) suggesting that it mainly affects cellular EMT. Moreover, co-electroporation of siRNA to Snail1 along with the full-length gene rescued the excessive myogenic differentiation caused by attenuation of Snail1 activity [see Additional file 8: Figure S8C,F,G], further substantiating the specificity of this loss of function approach.

Together, both gain and loss of function data implicate $\operatorname{Id} 2 / 3$, FoxC2 and Snail 1 as central factors regulating segregation of striated muscle versus SM progenitors from the lateral DM.

\section{Id2/3, FoxC2 and Snail1 integrate into a Muscle Regulatory Network}

Id 2 and Id 3 repress the myogenic function of Myf5 and activate FoxC2 transcription

$M y f 5$, a bHLH transcription factor, is expressed in the lateral DM of avian embryos prior to $M y o D$ [11]. Id2/3 are bHLH inhibitors whose repression promoted myogenesis
(Figure 3). Since Id2/3 are co-expressed with $M y f 5$, we examined whether the observed inhibition of myogenesis by the Id proteins can be explained by repression of Myf5 activity [33]. Over-expressing Myf5 induced precocious myocyte differentiation and this effect was completely abolished when Myf5 was over-expressed with either Id2 or Id3 (Figure 5A-D).

Furthermore, Id 2 or Id 3 induced FoxC2 mRNA, as exemplified in the central region of the DM that lacks endogenous transcription (Figure 5E-G). This function of Id proteins seemed to be independent of Id-Myf5 interactions, since over-expressing Myf5 in the lateral DM did not inhibit endogenous FoxC2 expression (Figure 5H,I). Thus, Id 2 and Id 3 have a two-pronged action, inhibiting the myogenic lineage by repressing Myf5 activity and up-regulating FoxC2 expression via a yet undefined mechanism.

\section{FoxC2 inhibits the myogenic program by repressing Pax7}

In the mouse, the balance of Foxc2 and Pax3/7 was shown to control vascular versus myotomal development, respectively [22]. We examined whether this balance operates similarly in the avian embryo. First, over-expression of Pax7 in the lateral DM promoted extensive myotome colonization and myogenic differentiation while inhibiting cell migration and $\mathrm{SM}$ production that was apparent under control conditions (Figure 6A,B). Since FoxC2 and Id2/3 displayed an opposite phenotype to that of Pax7 (Figures 3, 4, Additional files 6 and 7: S6, S7), we predicted they would negatively regulate Pax7. Indeed, FoxC2 and Id2 attenuated Pax7 expression in the DM, at the transcript and/or at the protein levels (Figure 6C-G and Additional file 9: Table S1). Conversely, Pax7 had no effect on FoxC2 or Id transcription [see Additional file 9: Table S1]. However, Pax7 repressed Snail1 mRNA and vice-versa, suggesting a negative feedback loop between the latter factors [see Additional file 9: Table S1].

Altogether, FoxC2, Id2/3 and Snail1 comprise a MRN that negatively influences the activity of distinct myogenic genes to favor SM at the expense of striated muscle development from the lateral DM.

\section{Notch signaling interacts with components of the MRN}

Since both Notch and the factors comprising the MRN were found to be necessary and sufficient for generating vascular fates, we examined possible interactions. In order to assess the possibility that Notch signaling affects expression of these factors, we turned to the central sheet of the $\mathrm{DM}$, the compartment closest to the lateral DM, in which these pro-SM factors are not endogenously expressed [see Additional file 3: Figure S3]. Indeed, aN2 was sufficient to ectopically upregulate $I d 2$, $I d 3$ and $F o x C 2$ mRNAs in this compartment of the DM 10 hours following transfection 

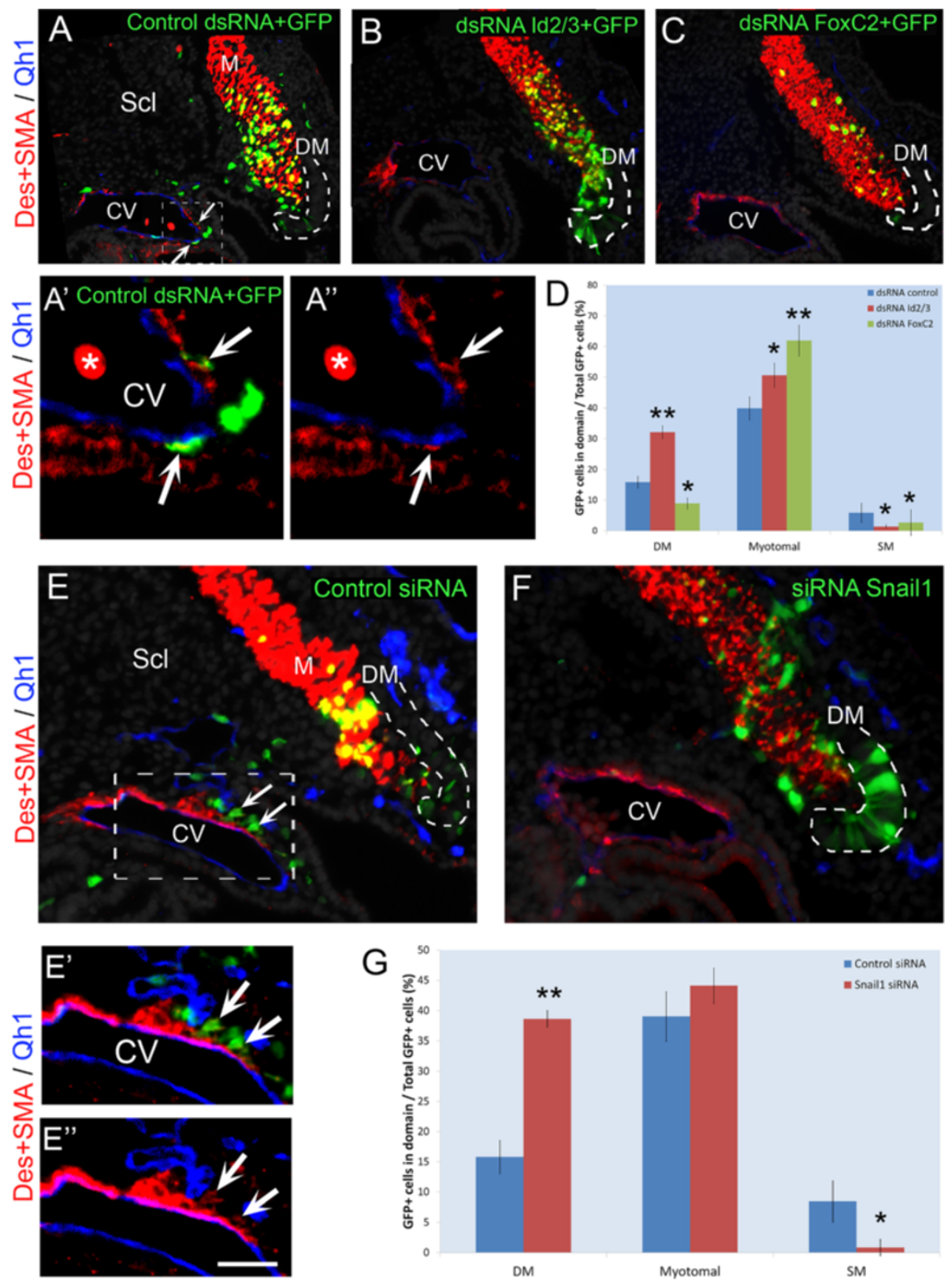

Figure 4 Id2, Id3, Foxc2 or Snail1 are necessary for development of vascular lineages. dsRNAs or siRNAs were electroporated into the presumptive lateral DM at E2 and embryos re-incubated for 40 hours. (A) dsRNA against LacZ was co-electroporated with a GFP serving as control $(\mathrm{N}=17)$. Note localization of labeled progeny in myotome $(M)$, ventral sclerotome (Scl) and cardinal vein (CV, arrows). ( $\left.\mathbf{A}^{\prime}, \mathbf{A}^{\prime \prime}\right)$ Magnification and channel breakdown of CV (dotted box in A). Arrows point to Desmin+/SMA + SM cells. (B) dsRNA to Id2/3 ( $N=5)$, (C) dsRNA to FoxC2 ( $N=16)$. Note the presence of labeled progeny in myotome and/or DM and their absence in blood vessels. (D) Quantifications of labeled cell distribution (mean \pm SEM, and see also Additional file 4: Figure S4B). (E) Control siRNA was electroporated as in A-C. Labeled progeny are both in myotome and cardinal vein $(C V)$ (arrows, $N=4)$. $\left(E^{\prime}, E^{\prime \prime}\right)$. Magnification and channel breakdown of $C V$ (dotted box in $E$ ). Arrows point to Desmin+/SMA + SM cells. (F) siRNA to Snail1 inhibits cell migration and SM development while labeled cells remain in the ventro-lateral lip of the DM ( $N=4)$.

Endothelial cells were visualized with the Qh1 antibody (blue). (G) Quantifications of labeled cell distribution (mean \pm SEM, and see also Additional file 4: Figure S4C). ${ }^{*} \leq 0.05 ;{ }^{* * P} \leq 0.01$ compared to controls. Lateral $D M$ is outlined with a dotted line. Bar: (A-C) $37 \mu m ;\left(\mathbf{A}^{\prime}, \mathbf{A}^{\prime \prime}\right) 15 \mu \mathrm{m} ;(\mathbf{E}, \mathbf{F})$ $50 \mu \mathrm{m} ;\left(\mathbf{E}^{\prime}, \mathbf{E}^{\prime \prime}\right) 25 \mu \mathrm{m}$. Des, desmin; DM, dermomyotome; dsRNA, double-stranded RNA; E, embryonic day; SEM, standard error of the mean; SMA, smooth muscle actin. 

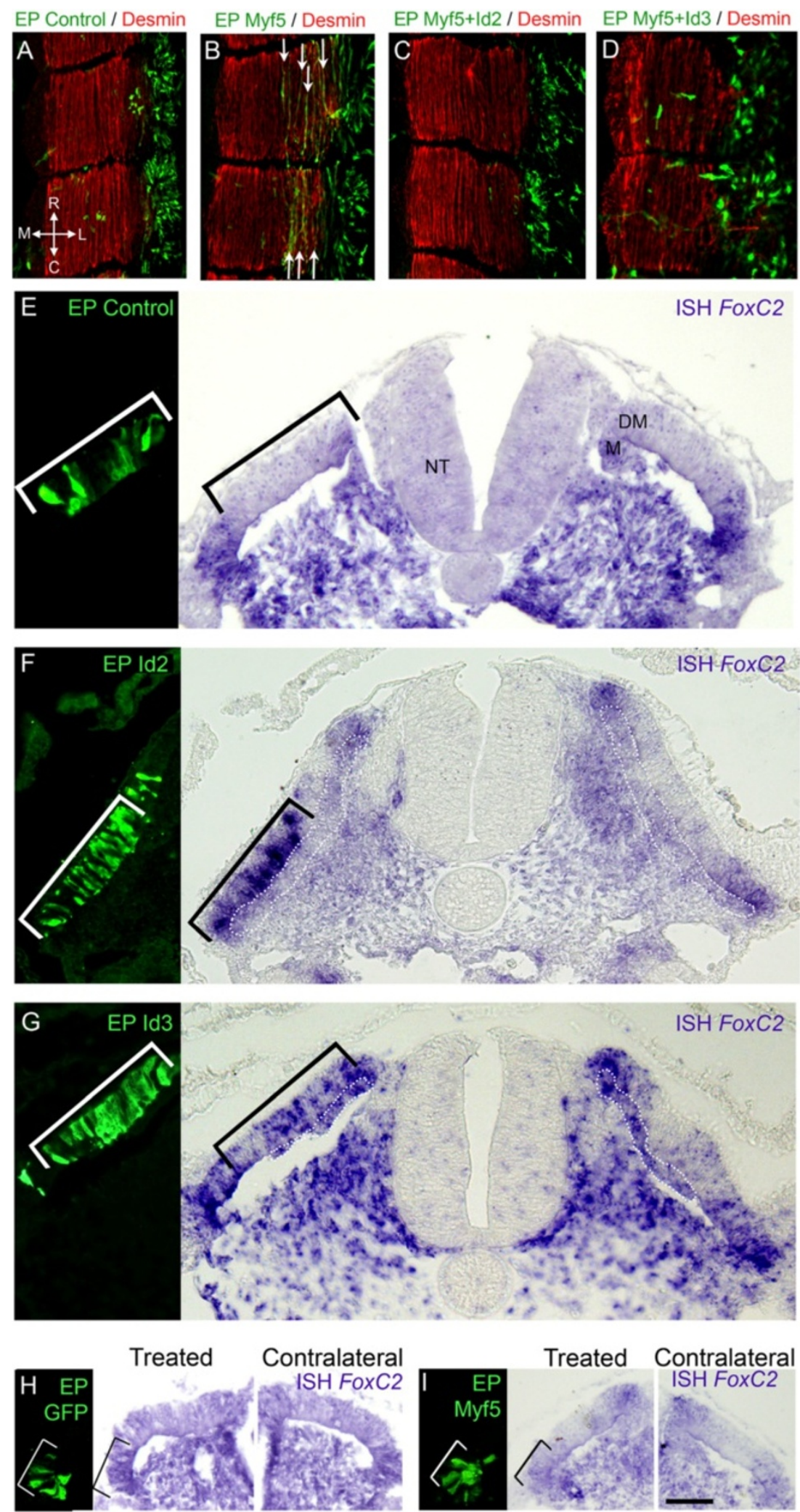

ISH FoxC2

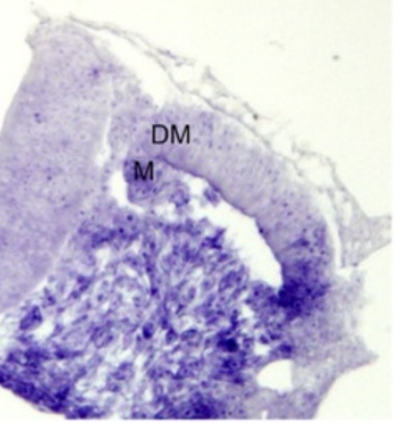

ISHFoxC2

ISH FoxC2
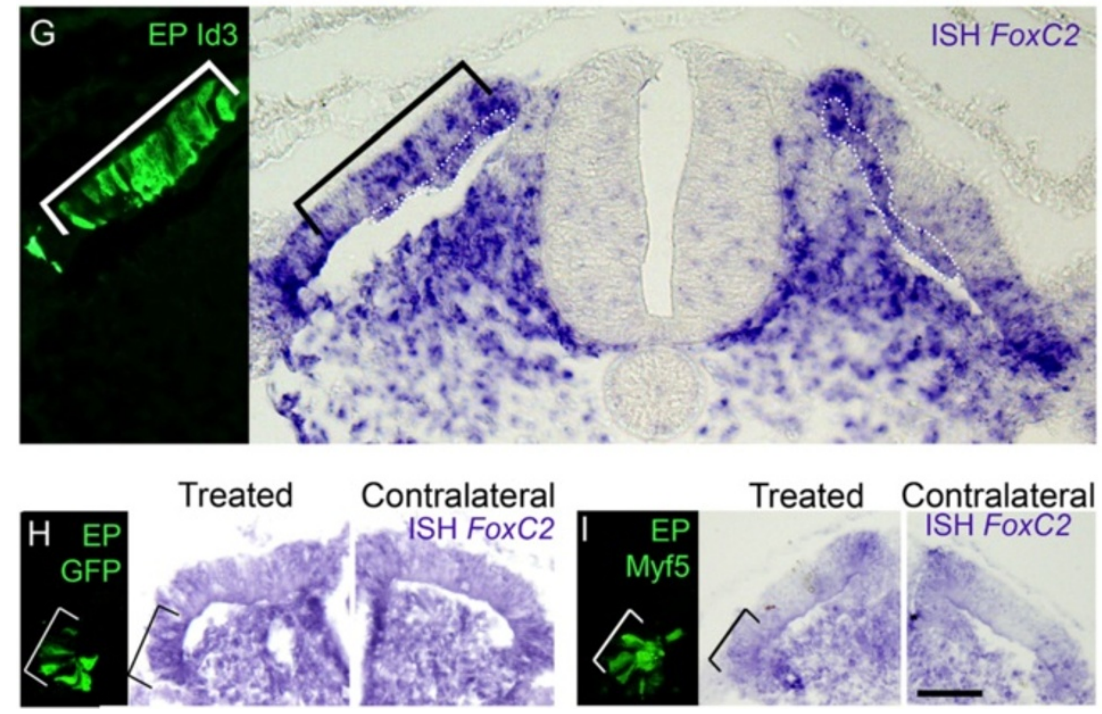

Figure $\mathbf{5}$ (See legend on next page.) 
(See figure on previous page.)

Figure $5 \mathrm{Id} / 3$ activity inhibits Myf5-induced myogenesis and upregulates FoxC2 transcription. (A) Electroporation of control GFP into the presumptive lateral DM at E2. At 16 hours post-treatment, labeled cells are still confined to the DM epithelium $(\mathrm{N}=3)$. (B) Missexpression of Myf5 induces premature fiber formation (arrows) $(\mathrm{N}=3)$. Co-treatment of Myf5 with Id2 $(\mathbf{C}, \mathrm{N}=3)$ or Id3 $(\mathbf{D}, \mathrm{N}=6)$ prevents Myf5-induced myogenesis and cells appear scattered instead of epithelial. (E-G) shows 12 hours of control-GFP $(\mathbf{E}, N=6)$, Id2 $(\mathbf{F}, N=9)$ or Id3 $(\mathbf{G}, N=7)$ missexpression in the central DM (left panels). Id2 or Id3 induce transcription of FoxC2 compared to the contralateral sides and to control-GFP. (H-I) Myf5 does not affect FoxC2 transcription. Control-GFP $(\mathbf{H}, \mathrm{N}=2)$ or Myf5 $(\mathbf{I}, \mathrm{N}=13)$ for 12 hours did not affect transcription of FoxC2 in the lateral DM. Bar: (E-G) $25 \mu \mathrm{m}$; (H) $40 \mu \mathrm{m}$; (I) $50 \mu \mathrm{m}$. DM, dermomyotome; EP, electroporation; NT, neural tube.
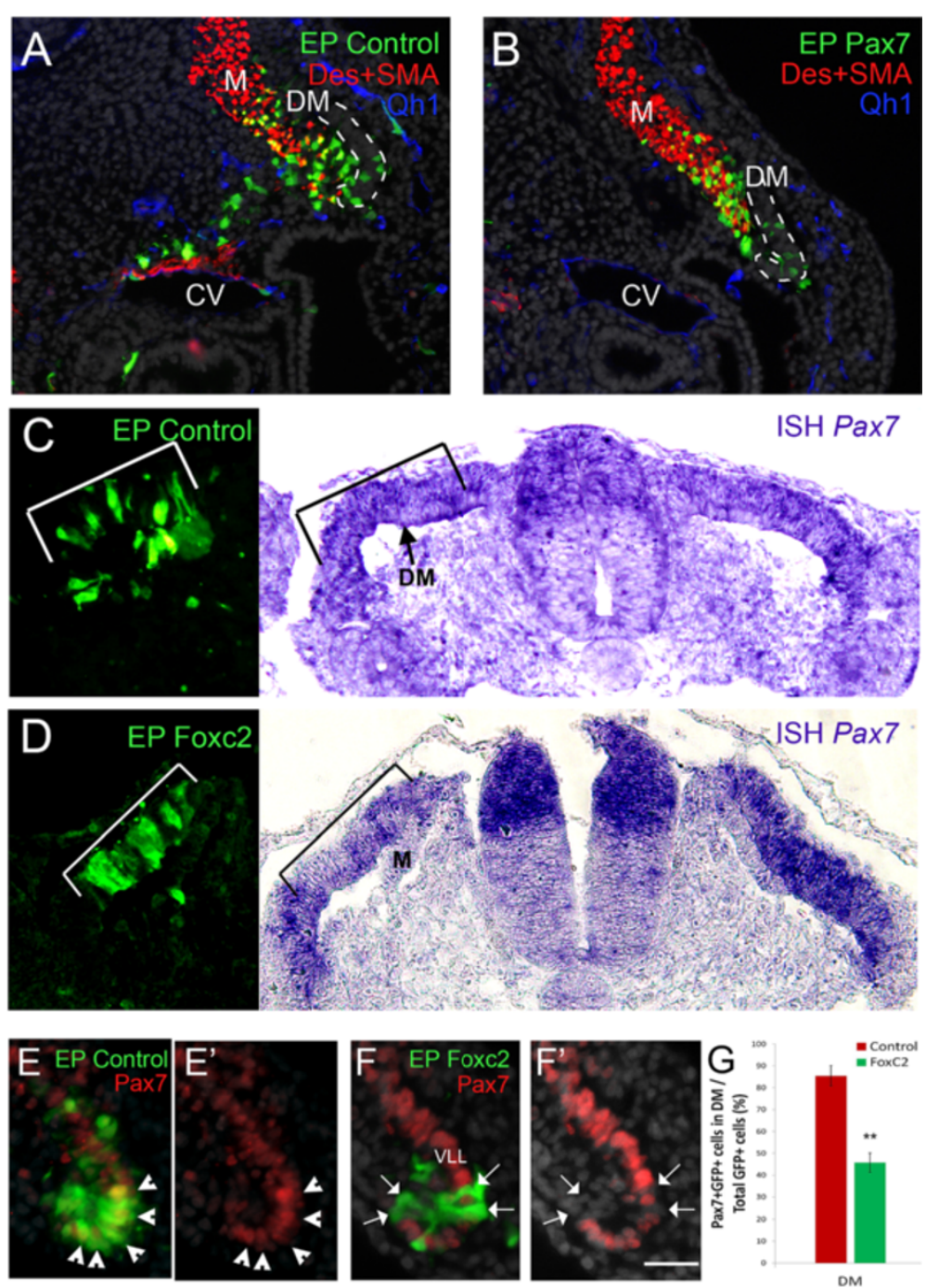

Figure 6 Foxc2 downregulates Pax7 that stimulates myotome colonization. (A) Control GFP alone or pMIW-Pax7/GFP (B) were electroporated as in Figures 1, 2 and 3 ( $N=7$ and 8, respectively). At 43 hours post-treatment control cells are present in myotome (M), sclerotome and wall of the cardinal vein (CV). In contrast, Pax7+ cells are restricted to the myotome where they co-express desmin. Endothelial cells were visualized with the Qh1 antibody (blue). (C-G) Foxc2 represses Pax7 expression. (C) Control-GFP ( $=5$ ). (D) Foxc2 was electroporated into the DM (left panel). Marked attenuation of Pax7 expression was detected at the mRNA (right panel, black bracket, $N=4$ ) compared to the contralateral side and to GFP-control (C). (E-G) GFP-control labeled cells exhibit Pax7 immunoreactivity (E, G, arrowheads, $N=7$ ), whereas fewer cells displayed Pax7 immunoreactivity following treatment with Foxc2 ( $\mathbf{F}, \mathbf{G}$, arrows, $\mathrm{N}=7$ ). (G) Quantification (mean $\pm \mathrm{SEM}$ ) of cells co-expressing Pax7 protein and FoxC2-GFP. ${ }^{*} P \leq 0.01$. Bar: (A-B) $30 \mu \mathrm{m} ;(\mathbf{C}, \mathbf{D}) 60 \mu \mathrm{m}, \mathbf{( E , F )} 25 \mu \mathrm{m}$. Des, desmin; DM, dermomyotome; EP, electroporation; ISH, in situ hybridization; SEM, standard error of the mean; SMA, smooth muscle actin; VLL, ventro-lateral lip. 
compared to the contralateral sides and to GFP-controls (Figure 7A-C, Additional file 10: S9).

Next, we examined whether there is feedback modulation of the factors on Notch signaling. To this end, we implemented a Notch reporter construct, comprising the mHes1 promoter driving GFP expression [34]. An avian homologue of Hes1, hairy2, is indeed expressed in the lateral DM [14], signifying active Notch signaling in this compartment. Id2 and Id3 ectopically enhanced reporter activity indicating that it augments Notch signaling (Figure 7D-F and see [34]; $2.7 \pm 0.6$ and 3.54 \pm 0.2 -fold for Id 2 and Id 3 over control values, $\mathrm{N}=6$ and 5 , $P<0.01$ and $P<0.05$, respectively). Thus, a positive feedback is formed, whereby Notch signaling activates $I d$ expression and Id in turn strengthens Notch signaling. Interestingly, no marked increase of Notch signaling was monitored in response to FoxC2 up-regulation [see Additional file 9: Table S1], yet Pax7 was found to silence reporter activity (Figure 7D,G, $0.16 \pm 0.9$-fold compared to control, $\mathrm{N}=7, P<0.01$ ). Thus, although FoxC2 does not enhance Notch signaling, by inhibiting Pax7 (Figure 6C-G) it presumably permits a basal level of Notch activation.

In addition, over-expression of Snail 1 rapidly promoted cell delamination from the lateral DM (Figure 3, Additional file 5: S5), whereas loss of Snail1 activity for 40 hours had the opposite effect (Figure 4 and Additional file 8: Figure S8). Since Notch similarly promoted EMT (Figure 1), we sought to examine whether Notch triggers cell delamination independently of Snail1 activity. A 16-hour exposure to aN2 promoted delamination and migration of the labeled cells when compared to the control GFP-treated epithelium (Figure 7H,I and Figure 1). In contrast, silencing Snail1 repressed cell delamination (Figure 7J, 4 F-G) and aN2 missexpression was unable to rescue this phenotype (Figure 7K). However, aN2 did not up-regulate Snail1 transcription in the DM [see Additional file 9: Table S1]. Thus, in lateral DM progenitors, the endogenous activity of Snail1 is essential for Notch-induced EMT.

\section{Positive regulation of the MRN by BMP signaling}

BMP4, secreted from the LPM, signals the nearby lateral DM to promote vascular development and inhibit terminal muscle differentiation $[11,14,15]$. To test whether BMP regulates $I d 2$, Id3, FoxC2 and Snail1, the secreted BMP antagonist noggin was electroporated into the LPM. Inhibition of BMP signaling completely abolished expression of $I d 2, I d 3, F o x C 2$ and Snail1 in the adjacent lateral DM compared to the contralateral intact sides (Figure 8A-D), demonstrating that BMP signaling is necessary for maintaining transcription of these factors.

We next asked whether BMP modulates Notch signaling. Electroporation of a BMP4-encoding plasmid substantially activated the GFP signal from the Hes1 promoter (Figure 8E,F). Reciprocally, aN2 enhanced BMP activity, as monitored by expression of a specific BMP reporter, BRE::

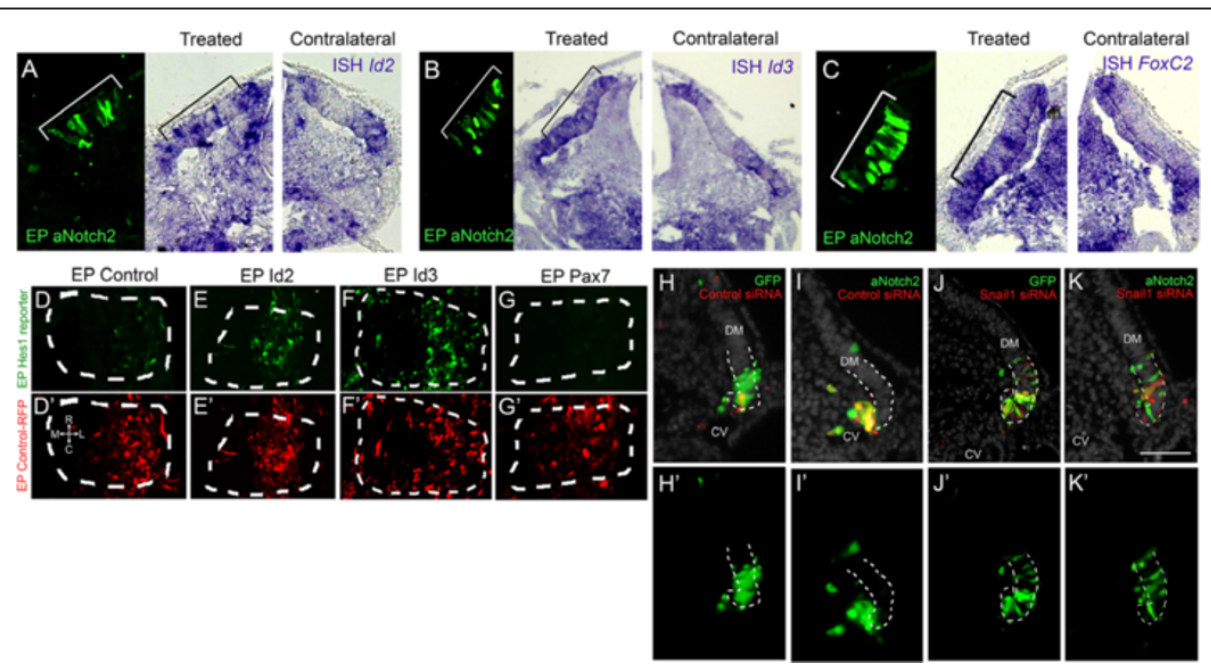

Figure 7 Interactions of Notch with factors of the MRN. (A-C) aNotch2 expression in the central DM (brackets, left panels) promotes transcription of Id2 (A, N = 4), Id3 ( $\mathbf{B}, \mathbf{N}=2)$ and FoxC2 (C, N =3) compared to the contralateral sides. (D-G) Id2, Id3 and Pax7 affect Notch signaling. Dorsal views of whole-mount segments. (D) Basal activity of Hes1 reporter in the lateral $D M(N=21)$. Id2 (E) or Id3 (F) missexpression enhances Hes1 reporter activity ( $N=13$ and 8, respectively), whereas Pax7 inhibits signaling $(\mathbf{G}, N=13)$. (D'-G') Control RFP shows similar electroporation efficiency in all treatments. (H-K) Notch-induced EMT requires Snail1 activity. $\left(\mathbf{H}, \mathbf{H}^{\prime}\right)$ Cells co-electroporated with control-GFP and control scrambled siRNA are mostly found within the lateral DM with a few cells delaminating from the epithelial sheet 16 hours post-treatment $(N=7)$. $\left(\mathbf{I}, \mathbf{I}^{\prime}\right)$ aNotch2 induces labeled cells to undergo EMT and migrate towards blood vessels $(N=6)$. $\left(\mathbf{J}, \mathbf{J}^{\prime}\right)$ Snail1 knock-down maintains cells within the lateral DM, inhibiting delamination $(\mathrm{N}=5)$. ( $\left(\mathbf{K}, \mathbf{K}^{\prime}\right)$ Snail1 knock-down hinders Notch-induced EMT, as transfected cells remain epithelial

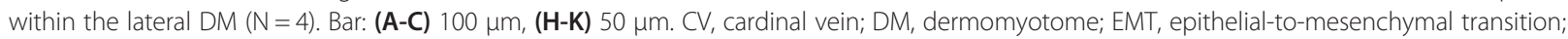
EP, electroporation; ISH, in situ hybridization; RFP, red fluorescent protein. 

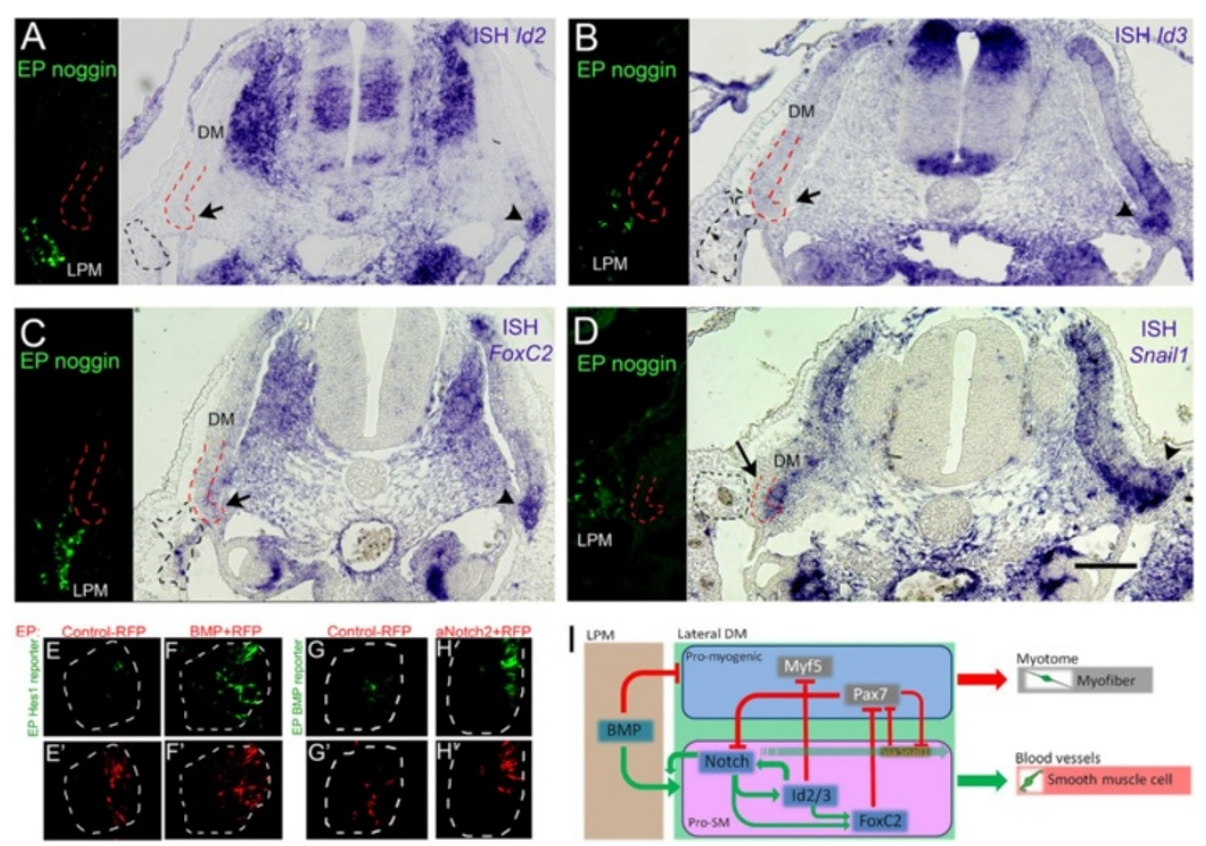

Figure 8 BMP regulates the vascular-promoting factors and communicates with Notch. (A-D) Ectopic noggin expression in the lateral plate mesoderm ( $(\mathrm{PPM}$, left panels) silences expression of Id2 ( $\mathbf{A}, \mathrm{N}=3)$, Id3 $(\mathbf{B}, N=3)$, Fox C2 $(\mathbf{C}, N=4)$ and Snail $(\mathbf{D}, N=2)$ in the lateral DM (arrows) compared to the contralateral sides (arrowheads). (E-H) Dorsal view of whole-mount segments. (E) Basal activity of Hes1 reporter in the lateral DM $(N=5)$. (F) Ectopic BMP expression enhances Hes1 reporter activity $(N=6)$. (G) Basal activity of the BMP reporter $(N=3)$. $(\mathbf{H})$ aNotch2 induces BMP reporter activity $(\mathrm{N}=7)$. $\left(\mathbf{E}^{\prime}-\mathbf{H}^{\prime}\right)$ Control RFP shows similar electroporation efficiency in all treatments. (I) A gene regulatory network underlying lineage segregation in the lateral DM. BMP from the LPM positively regulates Notch activity and vice-versa. Notch/BMP signaling upregulate/maintain expression of $1 d 2 / 3$ and FoxC2 which, in turn, promote lateral DM cells to delaminate, migrate and differentiate into SM in blood vessels (BV) at the expense of skeletal muscle, partly by inhibiting Myf5 and Pax7 activities. Furthermore, Notch-dependent cellular EMT is mediated by Snail1 and Pax7 inhibits Notch signaling and Snail1 transcription. In addition, Id2/3 enhance Notch signaling and vice-versa and Id2/3 upregulate FoxC2 mRNA, altogether reinforcing the network. Bar: (A-D) $100 \mu \mathrm{m}$. BMP, bone morphogenetic protein; DM, dermomyotome; EMT, epithelialto-mesenchymal transition; EP, electroporation; ISH, in situ hybridization; LPM, lateral plate mesoderm; M, myotome; RFP, red fluorescent protein; SM, smooth muscle.

GFP [35] (Figure 8G,H). Hence, a positive regulatory relationship exists between these two signaling pathways in the hypaxial region of the DM. Collectively, the data presented provide evidence for the existence of a regulatory network underlying lineage segregation from the lateral DM (Figure 8I).

\section{Discussion}

We identified Id2/3, FoxC2 and Snail1 as essential effectors of the balance between myogenic and SM fates in the lateral DM. Furthermore, we characterized some of their interactions in the context of myogenesis and their relationship with the Notch and BMP pathways, previously reported to affect this balance [14]. Together, we uncovered a MRN responsible for lineage segregation in the lateral DM (Figure 8I).

The current model suggests that early BMP from the LPM [36] triggers Notch signaling in the lateral DM. By a mutual positive interaction of these two signaling cascades, both pathways are stabilized and strengthened. Consequently, Id $2 / 3$ expression is upregulated, on the one hand inhibiting myogenesis by blocking Myf5-dependent myogenesis and inducing a delaminating program by a concomitant upregulation of FoxC2. FoxC2, in turn, represses Pax7, thereby preventing myogenesis and triggering instead cell delamination. Reduction of Pax7 protein relieves its inhibition on Snail 1 expression, the latter now mediating Notch-dependent EMT. Altogether this MRN ultimately leads to induction of the SM fate (Figure 8I).

Single cell lineage analysis in ovo suggested that while the early lateral epithelium generates striated and SM fates, the mature lateral DM mainly produces striated muscle [14]. Id2/3, FoxC2 and Snail1 are predominantly transcribed at the early stage, when they are likely to prevent premature striated myogenesis while promoting SM fates, in line with our previous results suggesting the existence of bi-fated $\mathrm{SM}$ /striated muscle progenitors in the lateral DM, whose definitive destiny is affected by manipulation of Notch signaling [14]. The timely downregulation of the above factors may, therefore, be necessary to transit toward the main myotomal phase issued from the lateral DM. 
The various genes in the network may affect distinct processes while converging to generate the SM fate. We noticed that both FoxC2 and Snail1 promoted a rapid EMT of lateral DM progenitors, a prerequisite for the generation of cellular migration through the sclerotome on the way to blood vessels. Both genes were indeed implicated in EMT in several contexts (see Introduction). EMT may, therefore, be part of the program that represses striated myogenesis $[37,38]$. Along this line, Pax7 that promoted myotome colonization is known to preserve epithelial integrity [39], and in the lateral DM FoxC2 inhibited Pax7 expression and Pax7 repressed Snail1 (our study). Consistently, FoxC2 was also shown to repress Pax3/7 in the mouse mesoderm [22]. Furthermore, Notch promotes EMT during cardiac development and oncogenesis via transcriptional activation of Snail1 [40]. In the DM, we show that Notch also promotes cell delamination only when endogenous Snail 1 is active but seems not to affect its level of transcription, and Pax7 inhibited Notch activity in agreement with its pro-myogenic effect. Along this line, we also show that Notch induces transcription of FoxC2, yet the latter had no reciprocal effect on Hes1 reporter activity. In endothelial cells, FoxC transcription factors were shown to regulate Hey2 and Dll4 mRNAs directly through interactions with the Notch intracellular domain [25]. Altogether, our data indicate that the control of the epithelio-mesenchymal status of progenitors may be an important level of regulation of cell fate decisions in the embryonic DM context.

We find that Id 2 and Id3, both present in the lateral DM, inhibit Myf5-dependent myogenesis. In satellite cells, Id3 was reported to be a direct transcriptional target of Pax7 and may also act in that context to inhibit MRF activity that results in sustained cell proliferation [41] and inhibition of myogenesis [42]. Furthermore, Notch upregulates $I d$ transcription in the DM (this study). The fact that Hairy2 is transcribed in the lateral DM [14] suggests that Notch signaling is active in this domain. This is consistent with data showing that Notch downstream effectors of the Hes family, as well as Dll4, upregulate Id expression which, in turn, compromise Myf5 activity $[33,43,44]$. Interestingly, Id expression, as well as the other network components, is also modulated by BMP that inhibits $M y o D$ and consequent muscle differentiation from the lateral somite [11] while promoting endothelial development [14]. Likewise, in cranial neural crest, Id2a is a target of BMP signaling that affects the choice between neural and skeletal muscle fates [45] and in the $\mathrm{C} 2 \mathrm{C} 12$ muscle cell line Id 2 was found to be a direct BMP target [46]. Thus, in the lateral somite and DM, Notch and BMP activities converge to positively regulate the network. Moreover, these signaling factors sustain each other's activities, as observed in other systems [47,48]. We propose that BMP operates upstream to control the timely development of vascular and skeletal muscle lineages as it is produced in the adjacent LPM and intermediate mesoderm at high levels during the somite and early DM stages and is subsequently downregulated $[11,36]$. This coincides with the downregulation of Id2/ 3, FoxC2 and Snail1 (this study) and with the advent of the main phase of skeletal myogenesis [14].

Growing evidence suggests that the DM is a heterogeneous epithelium with progenitors bearing different states of commitment and generating distinct fates and lineage combinations $[49,50]$. In this respect, a given signaling factor could potentially elicit different effects along the DM. Indeed, transient Notch signaling in the medial DM was found to trigger myogenesis [51] whereas in the lateral DM, although short exposure to Notch enabled myotome homing, myogenic differentiation did not proceed further. Upon longer exposure, lateral progenitors generated SM [this study and [14]]. Consistent with our findings, specific Notch missexpression in the central DM area of the mouse DM also stimulated SM/pericyte development in addition to maintaining progenitor cell properties [52]. Hence, different DM regions may differentially respond to Notch, perhaps due to their dissimilar microenvironment that leads to expression of distinct downstream genes in either domain.

Altogether, our results corroborate the notion that the Notch pathway, active in the lateral DM, promotes generation of SM at the expense of striated muscle [14]. This choice is also apparent during blood vessel consolidation, as endothelial cells via Dll4 and PDGF-BB promote the pericyte fate even in committed myoblasts [33]. Likewise, Notch regulates SM development in the heart epicardium [53] and is required for SMA expression in SM cells via activation of its effector CSL [54]. In the present context, it is possible that in progenitors of the SM lineage Notch signaling is activated twice with a cumulative effect; first in the lateral DM itself and then in the target blood vessels.

Taken together, the concerted activity of Notch/BMP and downstream genes of the network here presented may not merely prevent cell differentiation and maintain the progenitor state, but rather actively modulate cell fate choices in the DM.

\section{Conclusions}

By characterizing a molecular regulatory network responsible for the segregation of striated and smooth muscle from progenitors in the lateral dermomyotome, our data provide further support to the notion that lineage segregation depends on the integration of specific inputs within a cell. The various genes identified form a molecular network in which competing factors vie to induce a lineage identity on the cell. Factors within the network, such as FoxC2, Snail 1 and $\operatorname{Id} 2 / 3$, promote smooth muscle development by 
contending against pro-myotomal factors (Myf5 and Pax7) that promote a striated muscle fate (Figure 8I). The former are positively regulated by Notch signaling that influences several aspects of the process depending on signal duration, from inhibition of striated muscle differentiation to stimulation of cell delamination and, finally, promotion of a smooth muscle fate.

\section{Methods}

\section{Embryos}

Fertile quail (Coturnix coturnix japonica) or chicken (Gallus gallus) eggs from commercial sources (Moshav Mata and Moshav Orot, respectively) were used.

\section{Embryo processing and in situ hybridization}

Embryos were fixed with either 4\% formaldehyde or Fornoy, processed for paraffin wax embedding and sectioned at 8 or $10 \mu \mathrm{m}$ for immunostaining or in situ hybridization (ISH), respectively. The following probes were used: Id2 (full length), Id3 (full length), FoxC2 (268 to 662 of cFoxC2 cDNA), Snail1 (A. Nieto) and Pax7 (D. Duprez).

\section{Expression vectors and in ovo electroporation}

The following expression vectors were employed: cId2 and cId3, mFoxc2 [22], Snail1 (A. Nieto; [55]), the intracellular domain of Notch2 (aN2, S. Chiba; [56]), pMIW-Pax7 (H. Nakamura), pCA $\beta$-mMyf5-EGFP (D. Duprez), pCAGGqDIl1-IRES-GFP and pEFBOS-mBMP4 (Y. Wakamatsu), xNoggin [57] and pTRE2-dnMAML1-Flag-IRES-hrGFP (J. Jenssen; [58]). pCAGGS-AFP [59] and pRFPRNAiC were used as controls. For transient over-expression, plasmids were sub-cloned into pBI-TRE-EGFP. Each plasmid was co-electroporated with the pRev-Tet-off [60] at a 2:1 ratio and doxycycline $(300 \mu \mathrm{l}$ of $0.1 \mu \mathrm{g} / \mu \mathrm{l}$ administered every 12 hours) was added in order to silence plasmid transcription. For the Hes1 reporter experiments constructs of pBI-TRE-cId2/cId3/mFoxc2 were used following excision of the GFP cassette.

For knock-down experiments, dsRNA against Id2 (full length), Id3 (full length) and FoxC2 (268 to 662 from cDNA) were applied. dsRNA directed against $L a c Z$ was used as a control. Human FOXC2-Engrailed (A. MacDougald; [61]) was subcloned into pCAGG (pCAGG-FOXC2-EngrailedIRES-GFP). Two siRNA sequences against Snail1 were synthesized [28] and cloned into pRFPRNAiC [62]. A scrambled siRNA was used as a control.

To assay for Notch activity, the mHes1 promoter driving GFP expression in the ptk plasmid was used (N. Jing; [34]). The specificity of the Hes1 reporter to changes in Notch signaling was assessed with pBI-TRE-aN2 and pmiw-FLAG-cNumb [63].

To determine that Dll1 silences Notch activity, pCAGGDll1 (from Y. Wakamatsu) was introduced with the Hes1 reporter into HEK293 cells (see below). To assay for cell-autonomous silencing of Notch signaling by Dll1, pCAGG-Dll1-RFP was co-electroporated with pTP1 Venus, a CSL-dependent Venus (a GFP variant) reporter that carries 12 repeats of CSL-binding sites and that efficiently reflects Notch signaling activity [64].

To assay for BMP activity a BMP-responsive element driving GFP in the ptkh3 plasmid was used (E. Marti; [35]). Specificity of the BMP reporter was verified by coelectroporation with pEFBOS-mBMP4.

Electroporations were performed under a dissecting microscope. DNA (2 to $4 \mathrm{mg} / \mathrm{ml}$ ) was microinjected into the center of flank-level epithelial somites. Electroporations to the central DM, lateral DM or LPM were as described $[11,14,37]$. A square wave electroporator (ECM830, BTX, San Diego, CA, USA) was used to deliver one pulse of current at 15 volts, $10 \mathrm{~ms}$.

\section{Bromodeoxyuridine}

BrdU (140 $\mu$ l of a $10 \mathrm{mM}$ solution; Sigma-Aldrich, Rehovot, Israel) was applied for one hour eight hours postelectroporation, followed by fixation in $4 \%$ formaldehyde and processing for paraffin sectioning. Embryos were sectioned at $5 \mu \mathrm{m}$, and immunolabeled with anti-BrdU.

\section{Immunohistochemistry and image processing}

Antibodies used were against GFP (1:500; Invitrogen, Life Technologies, Grand Island, NY, USA), quail endothelial marker Qh1 (1:10) and Pax7 (1:10) (both from Developmental Studies Hybridoma Bank, University of Iowa, Iowa City, IA, USA), desmin (1:100; MP Biomedicals, Santa Ana, California, USA) and SMA (1:200; Sigma-Aldrich, Rehovot, Israel), both recognizing smooth and striated muscle [65], and ZO-1 (1:100, Zymed, Life Technologies, Grand Island, NY, USA). Secondary antibodies were coupled either to Cy2 or Rhodamine (1:100; Jackson ImmunoResearch Laboratories, West Grove, PA, USA). Nuclei were visualized with Hoechst.

Images were photographed using a DP70 (Olympus) cooled CCD digital camera mounted on a BX51 microscope (Olympus) [14]. Confocal sections of whole-mount preparations encompassing their entire thickness were photographed using a Nikon Eclipse 90i microscope with a Plan Apo 10x/ 0.45 dry objective (Nikon) and a D-Eclipse c1 confocal system (Nikon) at $2.7 \mu \mathrm{m}$ increments through the z-axis. Images were z-stacked with EZ-C1 3.90 FreeViewer software. For figure preparation, images were exported into Photoshop CS3 (Adobe). If necessary, the levels of brightness and contrast were adjusted to the entire image and images were cropped without color correction adjustments or $\gamma$ adjustments. Final figures were prepared using Photoshop CS2.

\section{Cell culture, DNA transfection and image analysis}

HEK293 cells were maintained in Dulbecco's modified Eagle's medium containing $10 \%$ fetal calf serum, D-glutamate and $1 \%$ penicillin and streptomycin. All the above were 
from Biological Industries (Biological Industries, Kibbutz Beit-Haemek, Israel). The Hes1-GFP reporter construct was introduced either alone or with pCAGG-Dll1 using polyethylenimine (PEI). A red fluorescent protein (RFP)-expressing plasmid was also included in each treatment to assay for transfection efficiency. Twenty hours after transfection, the fluorescence intensity of the GFP and RFP channels was measured using Photoshop CS3 $(\mathrm{N}=3$ random fields per plate, 3 plates per treatment). Hes1 activity was calculated as the ratio between the intensity of the green and red channels. Quantification represents mean \pm SEM.

\section{Data analysis}

To quantify the fate of lateral DM lineages, GFP + cells with visible Hoechst + nuclei were counted and classified according to marker expression and position. A total of 3 to 26 embryos were monitored per treatment; 3 to 14 or 5 to 15 sections/somite and 10 to 280 or 40 to 400 cells/ somite were counted at 20 hours or 40 hours, respectively. The proportion of a given derivative was calculated as the number of labeled cells out of the total number of GFP + cells in all sections and expressed as mean \pm SEM. Significance of results was determined using one-way or twoway independent Mann-Whitney test, using SPSS v20. A $P$-value $\leq 0.05$ was considered significant.

\section{Additional files}

Additional file 1: Figure S1. Inhibition of Notch signaling induces myogenesis. (A) HEK293 cells were co-transfected with a Dll1-encoding plasmid, with the Hes1 reporter to assay for Notch activity and with a RFP plasmid to assess transfection efficiency. Results represent the mean \pm SEM of triplicate cultures (see Methods). DII1 significantly reduced Hes 1 promoter activity $\left.{ }^{*} P<0.05\right)$. (B-C) Confocal images of somites electroporated with Dll1 (red) and with pTP-1Venus, a CSL-dependent reporter that reflects Notch signaling activity (green). Cells expressing Dll1 do not activate this Notch reporter cell autonomously, but rather activate it in adjacent cells. (D-G) Electroporation of the lateral DM with control GFP (D-D"), dnMAML1 (E), or DII1 (F). (D) At 40 hours post-electroporation, cells transfected with control GFP were found in myotome (M), sclerotome (SCl) and as SM within blood vessel walls (arrows and higher magnification in $\mathrm{D}^{\prime}$ and $\left.\mathrm{D}^{\prime \prime}, \mathrm{N}=6\right)$. $(\mathrm{E}, \mathrm{F})$ dnMAML1 and DII 1 abrogate cell migration through the sclerotome and subsequent vascular development while promoting the myotomal lineage ( $N=4$ and 4 , respectively). Lateral $D M$ is outlined by dotted lines. (G) Quantification (mean \pm SEM). ${ }^{*} P \leq 0.05$. Bar: (D-F) $50 \mu \mathrm{m}$; (D', D") $35 \mu \mathrm{m}$. CV, cardinal vein; DM, dermomyotome; meso, mesonephros; SEM, standard error of the mean.

Additional file 2: Figure S2. Late activation of Notch in lateral DM-derived progenitors is not sufficient for upregulating SM markers while in the ventral sclerotome. $\left(A, A^{\prime}\right)$ A Tet-GFP-control plasmid was activated twenty hours post-electroporation to the lateral DM for a period of eight hours. Note that the majority of GFP + cells still reside in the lateral DM and only a few delaminated into the intermediate zone between DM and myotome or into the ventral sclerotome (arrows) $(N=9)$. $\left(B, B^{\prime}\right)$ A Tet-aN2-GFP plasmid was activated twenty hours post-electroporation to the lateral DM for a period of eight hours. Most cells delaminated from the lateral DM and are located in the myotome as desmin/SMA-negative cells (B') or in the ventral sclerotome (arrows) $(\mathrm{N}=8)$. In both control and Notch-treated cells located in the ventral sclerotome no ectopic SMA/desmin immunostaining is evident. Hoechst nuclear staining is in grey. The lateral DMs are outlined by a dashed white line. Bar: 50 um. CV, cardinal vein; DM, dermomyotome; M, myotome; Scl, sclerotome; SM, smooth muscle; SMA, smooth muscle actin.

Additional file 3: Figure S3. Expression profiles of Id2, Id3, FoxC2 and Snaill to developing somites. Id2 (A, E, I), Id $3(B, F, J)$, FoxC2 $(C, G, K)$ and Snaill $(D, H, L)$ are expressed in the presumptive lateral domain of epithelial somites at E2.0 (dashed lines and yellow arrows in A-D) and in the lateral DM at E2.5 (E-H, black arrows), but are downregulated at E3.5 in the dissociated DM (I-L, black arrows) although the VLL is still epithelial (dashed lines). Note additional sites of expression of some of the transcripts in sclerotome and in the dorsomedial lip of the DM. Bar: (A-D) $50 \mu \mathrm{m}$, (E-L) $100 \mu \mathrm{m}$. CV, cardinal vein; DM, dermomyotome; E, embryonic day; $M$, myotome; VLL, ventro-lateral lip.

Additional file 4: Figure S4. Quantification of labeled cells in sclerotome. (A) Overexpression of Id2, Id 3, Foxc2 and Snail1 enhances the proportion of cells encountered in the sclerotome. Refers to Figure 3. (B) Knockdown of $I d 2 / 3$ and FoxC2. dsRNA to $I d 2 / 3$ reduce the proportion of labeled cells in sclerotome. Refers to Figure 4A-D. (C) Knockdown of Snail1 reduces the proportion of labeled cells in sclerotome. Refers to Figure 4E-G. In all cases, GFP + cells in sclerotome were negative for desmin and smooth muscle actin. Results are expressed as mean \pm SEM. ${ }_{* * P} \leq 0.01$. dsRNA, double-stranded RNA; SEM, standard error of the mean.

Additional file 5: Figure S5. Short-term over-expression of Foxc2 or Snail1 enhance delamination of lateral dermomyotome (DM) progenitors. (A) Cells transfected with a control GFP plasmid are mainly located as epithelial cells within the lateral DM and some have translocated into the intermediate zone, between the ventro-lateral lip (VLL) and the myotome (M) 16 hours post-electroporation ( $N=12)$. (B) Foxc2 overexpression. Note depletion of cells from the DM and the presence of labeled cells in an intermediate zone and in the sclerotome $(S \mathrm{Cl})(\mathrm{N}=17)$. $\left(\mathrm{C}, \mathrm{C}^{\prime}\right)$ Control cells marked with GFP reside as epithelial cells within the lateral DM and some have translocated into the myotome (M) 16 hours post-electroporation; note the epithelial morphology of the lateral DM marked by ZO-1 (white bracket) $(N=5)$. ( $\left(D, D^{\prime}\right)$ Sixteen hours of enhanced Snail1 activity compromises the epithelial morphology of treated cells, as indicated by the absence of the epithelial marker ZO-1 (white bracket). These cells exit the epithelial sheet but fail to contribute to the myotome $(N=5)$. Solid and dashed lines demarcate DM and myotome, respectively. (E) Quantifications (mean \pm SEM) of over-night exposure to Foxc2 and Snail1. **P-value $\leq 0.01$. Bar: $50 \mu \mathrm{m}$. CV, cardinal vein; SEM, standard error of the mean.

Additional file 6: Figure S6. Attenuation of Id $2 / 3$ activities causes precocious differentiation of myocytes in myotome. (A-C) Dorsal views of whole mount segments. (A) Cells treated with control dsRNA against LacZ are still located within the lateral DM (arrows) 16 hours post-electroporation whereas cells that received dsRNA against Id2/3 had already differentiated into myofibers ( $B$, arrows). (C) Co-transfection of Id $2 / 3$ along with dsRNAs to Id $/ 3$ rescues the knock-down phenotype, as cells fail to differentiate into fibers (arrows) and instead migrate ventro-laterally (arrowhead). (D-D") Transverse section 40 hours post-electroporation showing that Id2/3 over-expression rescues the Id2/ 3 knock-down phenotype $(N=5)$. ( $\left(D^{\prime}, D^{\prime \prime}\right)$ High magnification of the inset in $D$ showing the cardinal vein exhibiting co-localization of a labeled cell with SM markers, desmin and SMA. Endothelial cells were visualized with the Qh1 antibody (blue). (E) Quantification (mean \pm SEM) of labeled cell distribution ( $N=16$ for control; $N=19$ for Id2/3; $N=5$ for dsRNA Id2/3; $N=5$ for dsRNA Id2/3 + ld2/3). ${ }^{*} P \leq 0.05 ;{ }^{* *} P \leq 0.01$. Bar: (D) $100 \mu \mathrm{m}$; $\left(D^{\prime}, D^{\prime \prime}\right) 12.5 \mu \mathrm{m}$. CV, cardinal vein; Des, desmin; dsRNA, double-stranded RNA; M, myotome; SEM, standard error of the mean; SMA, smooth muscle actin.

Additional file 7: Figure S7. FoxC2 knock-down induces myocyte differentiation at the expense of vascular fates. (A-B) Dorsal view of whole-mount segments electroporated with control-GFP (A) or with FOXC2-engrailed (En) (B) and incubated for 16 hours. Only a few elongating myocytes are observed in the control (arrows, $N=8$ ), whereas FOXC2-En promoted premature fiber formation (arrows in $B, N=6)$. $(C, D)$ Transverse sections 40 hours post-electroporation with (C) control-GFP or (D) FOXC2-En. In control embryos cells are apparent in both vascular (as SM cells, see $C^{\prime}$ and $\left.C^{\prime \prime}\right)$ and myotomal domains $(N=4)$. $\left(C^{\prime}, C^{\prime \prime}\right)$ High 
magnification and channel breakdown of the inset showing the cardinal vein (CV) exhibiting co-localization of a GFP-labeled cell with Des/SMA immunoreactivity (arrow). (D) In contrast, knocking-down FoxC2 promotes myotomal colonization while hindering development of SM cells $(N=5)$. (E) Quantification (mean $\pm S E M$ ) of labeled cell distribution. $(F, G)$ Dorsal view of whole-mount segments 16 hours post-electroporation of (F) control dsRNA LacZ or (G) dsRNA FoxC2. In control embryos cells are in the lateral DM (arrows, $\mathrm{N}=3$ ) whereas in the presence of dsRNA FoxC2 myotomal cells begun differentiating (arrows in $G, N=3)$. $(H)$ Transverse section showing that co-electroporation of full-length Foxc2 along with dsRNA FoxC2 rescues the FoxC2 knockdown phenotype by inducing cell migration through the sclerotome and SM fate (arrow, see $\mathrm{H}^{\prime}, \mathrm{H}^{\prime \prime}$ ) and lack of myotome colonization (compare to Figures $3 \mathrm{~A}, \mathrm{C}$ and $4 \mathrm{C})$. ( $\left.\mathrm{H}^{\prime}, \mathrm{H}^{\prime \prime}\right)$ High magnification and channel breakdown of the inset in $\mathrm{H}$ showing the $\mathrm{CV}$ exhibiting co-localization of a labeled cell with Des/SMA + immunoreactivity. Qh1 antibody (blue) labels endothelial cells. (I) Quantification (mean \pm SEM) of labeled cell distribution ( $N=22$ for control dsRNA; $N=16$ for Foxc2; $N=16$ for dsRNA FoxC2; $N=7$ for dsRNA FoxC2 + Foxc2). ${ }^{*} P \leq 0.05 ;{ }^{*} P \leq 0.01$. Bar: (C,D) $50 \mu \mathrm{m} ;(\mathrm{H}) 35 \mu \mathrm{m} ;\left(\mathrm{C}^{\prime}, \mathrm{C}^{\prime \prime}, \mathrm{H}^{\prime}, \mathrm{H}^{\prime \prime}\right) 17.5 \mu \mathrm{m}$. Des, desmin; dsRNA, double-stranded RNA; M, myotome; SEM, standard error of the mean; SMA, smooth muscle actin.

Additional file 8: Figure S8. Attenuation of Snail1 signaling promotes myocyte differentiation and retention of cells in the lateral epithelium. (A-C) Dorsal view of whole-mount segments. (A) A control scrambled siRNA was electroporated into the lateral somites. At 16 hours post-electroporation few labeled cells have differentiated into myofibers. Other cells, while still residing in the lateral DM have lost their epithelial morphology. (B) In the presence of siRNA to Snail1 both maintenance of transfected epithelial cells within the lateral DM as well as premature myogenesis are apparent. (C) Snail1 over-expression rescues the siRNA-Snail1 knock-down phenotype, as fewer fibers and more scattered cells are observed (arrow). (D-F) Transverse sections 40 hours post-electroporation. (D) Control scrambled siRNA. Labeled cells are distributed in myotome (M), sclerotome and approach the cardinal vein (CV). (E) Knocking-down Snail1 maintains cells within the lateral DM as epithelial cells. (F) Over-expression of Snail1 rescues the effect of siRNA-Snail1 knock-down. The lateral DM is depleted of labeled cells and a notable fraction is able to migrate towards target sites (arrows). $\left(F^{\prime}, F^{\prime \prime}\right)$ High magnification of the inset in $F$ showing the CV exhibiting co-localization of a labeled cell with SM markers. Endothelial cells were visualized with the Qh1 antibody (blue). (G) Quantification (mean \pm SEM) of labeled cell distribution ( $N=7$ for control; $N=7$ for Snail 1 ; $=6$ for siRNA Snail1; $N=3$ for Snail 1 + siRNA Snail1). ${ }^{*} P \leq 0.05 ;{ }^{*} P \leq 0.01$. Bar: (D-F) $50 \mu \mathrm{m},\left(F^{\prime}-F^{\prime \prime}\right) 32 \mu \mathrm{m}$. es, desmin; DM, dermomyotome; SEM, standard error of the mean; siRNA, small interfering RNA; SMA, smooth muscle actin.

Additional file 9: Table S1. Interactions between genes of the regulatory network. Genes depicted in the horizontal axis (bold) were electroporated and genes or reporters depicted along the vertical axis were analyzed for expression by in situ hybridization, immunohistochemistry or activity (see text for details).

Additional file 10: Figure S9. Control-GFP to the central sheet does not affect transcription of Id2, Id3, or FoxC2. (A-C) GFP was electroporated into the presumptive central sheet of E2.0 embryos and re-incubated for an additional 10 hours (left panels). No changes in transcription were detected by in situ hybridization with probes against Id2 (,$N=5)$, Id3 $(B, N=5)$ or FoxC2 $(C, N=6)$ compared to the contralateral sides. Bar: $50 \mu \mathrm{m}$. E, embryonic day.

\section{Abbreviations}

aN2: active Notch2; bHLH: basic helix-loop-helix; BMP: bone morphogenetic protein; BrdU: 5-bromo-2'-deoxyuridine; Des: desmin; DM: dermomyotome; dn: dominant-negative; dsRNA: double-stranded RNA; E: embryonic day; EMT: epithelial-to-mesenchymal transition; EP: electroporation; GFP: green fluorescent protein; ISH: in situ hybridization; LPM: lateral plate mesoderm; MRN: Muscle Regulatory Network; siRNA: small interfering RNA; SM: smooth muscle; SMA: smooth muscle actin; TF: transcription factor.

\section{Competing interests}

The authors declare that they have no competing interests.

\section{Authors' contributions}

MA, RBY and CK conceived the project. MA and RBY performed experiments and data analysis. MA and CK wrote the manuscript. All authors read and approved the final manuscript.

\section{Acknowledgements}

We thank Margaret Buckingham for advice and discussions. We are indebted to numerous colleagues for sharing reagents, Tallie Bdolach for assistance with statistics and all members of our group for discussions. This work was supported by grants from the DFG (UN34/27-1), Association Francaise contre les Myopathies (\#15642) and the Israel Science Foundation (\#11/09 and \#97/13) to CK.

\section{Author details}

'Department of Medical Neurobiology, IMRIC and ELSC, Hebrew University of Jerusalem-Hadassah Medical School, Jerusalem 9112102, P.O. Box 12272, Israel. ${ }^{2}$ Present address: Cardiovascular Research Center, MGH Harvard Medical School, Charlestown, MA 02129, USA.

Received: 27 April 2014 Accepted: 24 June 2014

Published: 12 July 2014

\section{References}

1. Arendt $D$ : The evolution of cell types in animals: emerging principles from molecular studies. Nat Rev Genet 2008, 9:868-882.

2. Buckingham M, Vincent SD: Distinct and dynamic myogenic populations in the vertebrate embryo. Curr Opin Genet Dev 2009, 19:444-453.

3. Shieh PB: Muscular dystrophies and other genetic myopathies. Neurol Clin 2013, 31:1009-1029.

4. Scaal M, Christ B: Formation and differentiation of the avian dermomyotome. Anat Embryol 2004, 208:411-424.

5. Christ B, Scaal M: Formation and differentiation of avian somite derivatives. Adv Exp Med Biol 2008, 638:1-41.

6. Vasyutina $\mathrm{E}$, Birchmeier $\mathrm{C}$ : The development of migrating muscle precursor cells. Anat Embryol (Berl) 2006, 211:37-41.

7. Buckingham M, Bajard L, Chang T, Daubas P, Hadchouel J, Meilhac S, Montarras D, Rocancourt D, Relaix F: The formation of skeletal muscle: from somite to limb. J Anat 2003, 202:59-68.

8. Kardon G, Campbell JK, Tabin CJ: Local extrinsic signals determine muscle and endothelial cell fate and patterning in the vertebrate limb. Dev Cell 2002, 3:533-545.

9. Yvernogeau L, Auda-Boucher G, Fontaine-Perus J: Limb bud colonization by somite-derived angioblasts is a crucial step for myoblast emigration Development 2012, 139:277-287.

10. Tozer S, Bonnin MA, Relaix F, Di Savino S, Garcia-Villalba P, Coumailleau P, Duprez D: Involvement of vessels and PDGFB in muscle splitting during chick limb development. Development 2007, 134:2579-2591.

11. Kahane N, Ben-Yair R, Kalcheim C: Medial pioneer fibers pattern the morphogenesis of early myoblasts derived from the lateral somite. Dev Biol 2007, 305:439-450.

12. Cinnamon Y, Kahane N, Kalcheim C: Characterization of the early development of specific hypaxial muscles from the ventrolateral myotome. Development 1999, 126:4305-4315.

13. Gros J, Scaal M, Marcelle C: A two-step mechanism for myotome formation in chick. Dev Cell 2004, 6:875-882.

14. Ben-Yair R, Kalcheim C: Notch and bone morphogenetic protein differentially act on dermomyotome cells to generate endothelium, smooth, and striated muscle. J Cell Biol 2008, 180:607-618.

15. Nimmagadda S, Geetha Loganathan P, Huang R, Scaal M, Schmidt C Christ B: BMP4 and noggin control embryonic blood vessel formation by antagonistic regulation of VEGFR-2 (Quek1) expression. Dev Biol 2005, 280:100-110.

16. Benezra R, Davis RL, Lockshon D, Turner DL, Weintraub H: The protein Id: a negative regulator of helix-loop-helix DNA binding proteins. Cell 1990, 61:49-59.

17. Hollnagel A, Oehlmann V, Heymer J, Rüther U, Nordheim A: Id genes are direct targets of bone morphogenetic protein induction in embryonic stem cells. J Biol Chem 1999, 274:19838-19845. 
18. Yokoyama S, Asahara H: The myogenic transcriptional network. Cell Mol Life Sci 2011, 68:1843-1849.

19. Mani SA, Yang J, Brooks M, Schwaninger G, Zhou A, Miura N, Kutok JL, Hartwell K, Richardson AL, Weinberg RA: Mesenchyme Forkhead 1 (FOXC2) plays a key role in metastasis and is associated with aggressive basal-like breast cancers. Proc Natl Acad Sci U S A 2007, 104:10069-10074.

20. Hollier BG, Tinnirello AA, Werden SJ, Evans KW, Taube JH, Sarkar TR, Sphyris N, Shariati M, Kumar SV, Battula VL, Herschkowitz Jl, Guerra R, Chang JT, Miura N, Rosen JM, Mani SA: FOXC2 expression links epithelial-mesenchymal transition and stem cell properties in breast cancer. Cancer Res 2013, 73:1981-1992.

21. Winnier GE, Hargett L, Hogan BL: The winged helix transcription factor $\mathrm{MFH} 1$ is required for proliferation and patterning of paraxial mesoderm in the mouse embryo. Genes Dev 1997, 11:926-940.

22. Lagha M, Brunelli S, Messina G, Cumano A, Kume T, Relaix F, Buckingham ME: Pax3:Foxc2 reciprocal repression in the somite modulates muscular versus vascular cell fate choice in multipotent progenitors. Dev Cell 2009, 17:892-899

23. Seo S, Fujita H, Nakano A, Kang M, Duarte A, Kume T: The forkhead transcription factors, Foxc1 and Foxc2, are required for arterial specification and lymphatic sprouting during vascular development. Dev Biol 2006, 294:458-470.

24. Kume $T$, Jiang $H$, Topczewska JM, Hogan BL: The murine winged helix transcription factors, Foxc1 and Foxc2, are both required for cardiovascular development and somitogenesis. Genes Dev 2001, 15:2470-2482

25. Hayashi $\mathrm{H}$, Kume T: Foxc transcription factors directly regulate DII4 and Hey2 expression by interacting with the VEGF-Notch signaling pathways in endothelial cells. PLoS One 2008, 3:e2401.

26. Barrallo-Gimeno A, Nieto MA: The Snail genes as inducers of cell movement and survival: implications in development and cancer. Development 2005, 132:3151-3161.

27. Nieto MA, Sargent MG, Wilkinson DG, Cooke J: Control of cell behavior during vertebrate development by slug, a zinc finger gene. Science 1994, 264:835-839.

28. Delfini MC, De La Celle M, Gros J, Serralbo O, Marics I, Seux M, Scaal M, Marcelle $C:$ The timing of emergence of muscle progenitors is controlled by an FGF/ERK/SNAIL1 pathway. Dev Biol 2009, 15:229-237.

29. de Celis JF, Bray S: Feed-back mechanisms affecting Notch activation at the dorsoventral boundary in the Drosophila wing. Development 1997, 124:3241-3251.

30. Miller AC, Lyons EL, Herman TG: cis-Inhibition of Notch by endogenous Delta biases the outcome of lateral inhibition. Curr Biol 2009, 19:1378-1383.

31. Sprinzak D, Lakhanpal A, Lebon L, Santat LA, Fontes ME, Anderson GA Garcia-Ojalvo J, Elowitz MB: Cis-interactions between Notch and Delta generate mutually exclusive signalling states. Nature 2010, 465:86-90.

32. Sato Y, Watanabe T, Saito D, Takahashi T, Yoshida S, Kohyama J, Ohata E, Okano H, Takahashi Y: Notch mediates the segmental specification of angioblasts in somites and their directed migration toward the dorsal aorta in avian embryos. Dev Cell 2008, 14:890-901.

33. Cappellari O, Benedetti S, Innocenzi A, Tedesco FS, Moreno-Fortuny A, Ugarte G, Lampugnani MG, Messina G, Cossu G: DII4 and PDGF-BB convert committed skeletal myoblasts to pericytes without erasing their myogenic memory. Dev Cell 2013, 24:586-599.

34. Bai G, Sheng N, Xie Z, Bian W, Yokota Y, Benezra R, Kageyama R, Guillemot F, Jing $N$ : Id sustains Hes 1 expression to inhibit precocious neurogenesis by releasing negative autoregulation of Hes1. Dev Cell 2007, 13:283-297.

35. Le Dreau G, Garcia-Campmany L, Rabadan MA, Ferronha T, Tozer S, Briscoe J, Marti E: Canonical BMP7 activity is required for the generation of discrete neuronal populations in the dorsal spinal cord. Development 2012, 139:259-268

36. Sela-Donenfeld D, Kalcheim C: Localized BMP4-noggin interactions generate the dynamic patterning of noggin expression in somites. Dev Biol 2002, 246:311-328.

37. Kahane N, Ribes V, Kicheva A, Briscoe J, Kalcheim C: The transition from differentiation to growth during dermomyotome-derived myogenesis depends on temporally restricted hedgehog signaling. Development 2013, 140:1740-1750.

38. Ben-Yair R, Kahane N, Kalcheim C: LGN-dependent orientation of cell divisions in the dermomyotome controls lineage segregation into muscle and dermis. Development 2011, 138:4155-4166.
39. Wiggan O, Fadel M, Hamel P: Pax3 induces cell aggregation and regulates phenotypic mesenchymal-epithelial interconversion. J Cell Sci 2003, 115:517-529.

40. Timmerman LA, Grego-Bessa J, Raya A, Bertran E, Perez-Pomares JM, Diez J, Aranda S, Palomo S, McCormick F, Izpisua-Belmonte JC, de la Pompa JL: Notch promotes epithelial-mesenchymal transition during cardiac development and oncogenic transformation. Genes Dev 2004, 18:99-115.

41. Diao Y, Guo X, Li Y, Sun K, Lu L, Jiang L, Fu X, Zhu H, Sun H, Wang H, Wu Z: Pax3/7BP is a Pax7- and Pax3-binding protein that regulates the proliferation of muscle precursor cells by an epigenetic mechanism. Cell Stem Cell 2012, 11:231-241.

42. Wang Q, Fang WH, Krupinski J, Kumar S, Slevin M, Kumar P: Pax genes in embryogenesis and oncogenesis. J Cell Mol Med 2008, 12:2281-2294.

43. Tapanes-Castillo A, Baylies MK: Notch signaling patterns Drosophila mesodermal segments by regulating the bHLH transcription factor twist. Development 2004, 131:2359-2372

44. Reynaud-Deonauth S, Zhang H, Afouda A, Taillefert S, Beatus P, Kloc M, Etkin LD, Fischer-Lougheed J, Spohr G: Notch signaling is involved in the regulation of Id 3 gene transcription during Xenopus embryogenesis. Differentiation 2002, 69:198-208.

45. Das A, Crump JG: Bmps and id2a act upstream of twist1 to restrict ectomesenchyme potential of the cranial neural crest. PLoS Genet 2012, 8:e1002710.

46. Nakahiro T, Kurooka H, Mori K, Sano K, Yokota Y: Identification of BMP-responsive elements in the mouse Id2 gene. Biochem Biophys Res Commun 2010, 399:416-421.

47. Dahlqvist C, Blokzijl A, Chapman G, Falk A, Dannaeus K, Ibanez CF, Lendahl U: Functional Notch signaling is required for BMP4-induced inhibition of myogenic differentiation. Development 2003, 130:6089-6099.

48. Blokzijl A, Dahlqvist C, Reissmann E, Falk A, Moliner A, Lendahl U, Ibanez CF: Cross-talk between the Notch and TGF-beta signaling pathways mediated by interaction of the Notch intracellular domain with Smad3. J Cell Biol 2003, 163:723-728.

49. Nitzan E, Kalcheim C: Neural crest and somitic mesoderm as paradigms to investigate cell fate decisions during development. Develop Growth Differ 2013, 55:60-78.

50. Kalcheim C, Ben-Yair R: Cell rearrangements during development of the somite and its derivatives. Curr Opin Genet Dev 2005, 15:1-10.

51. Rios AC, Serralbo O, Salgado D, Marcelle C: Neural crest regulates myogenesis through the transient activation of NOTCH. Nature 2011, 473:532-535.

52. Mourikis P, Gopalakrishnan S, Sambasivan R, Tajbakhsh S: Cell-autonomous Notch activity maintains the temporal specification potential of skeletal muscle stem cells. Development 2012, 139:4536-4548.

53. Grieskamp T, Rudat C, Ludtke TH, Norden J, Kispert A: Notch signaling regulates smooth muscle differentiation of epicardium-derived cells. Circ Res 2011, 108:813-823.

54. Noseda M, Fu Y, Niessen K, Wong F, Chang L, McLean G, Karsan A: Smooth Muscle alpha-actin is a direct target of Notch/CSL. Circ Res 2006, 98:1468-1470.

55. Morales AV, Acloque H, Ocana OH, de Frutos CA, Gold V, Nieto MA: Snail genes at the crossroads of symmetric and asymmetric processes in the developing mesoderm. EMBO Rep 2007, 8:104-109.

56. Shimizu K, Chiba S, Saito T, Takahashi T, Kumano K, Hamada Y, Hirai H: Integrity of intracellular domain of Notch ligand is indispensable for cleavage required for release of the Notch2 intracellular domain. Embo $\mathrm{J}$ 2002, 21:294-302.

57. Endo Y, Osumi N, Wakamatsu Y: Bimodal functions of Notch-mediated signaling are involved in neural crest formation during avian ectoderm development. Development 2003, 129:863-873.

58. Afelik S, Qu X, Hasrouni E, Bukys MA, Deering T, Nieuwoudt S, Rogers W, Macdonald RJ, Jensen J: Notch-mediated patterning and cell fate allocation of pancreatic progenitor cells. Development 2012, 139:1744-1753.

59. Momose T, Tonegawa A, Takeuchi J, Ogawa H, Umesono K, Yasuda K. Efficient targeting of gene expression in chick embryos by microelectroporation. Dev Growth Differ 1999, 41:335-344.

60. Sato Y, Kasai T, Nakagawa S, Tanabe K, Watanabe T, Kawakami K, Takahashi Y: Stable integration and conditional expression of electroporated transgenes in chicken embryos. Dev Biol 2007, 305:616-624. 
61. Gerin I, Bommer GT, Lidell ME, Cederberg A, Enerback S, Macdougald OA: On the role of FOX transcription factors in adipocyte differentiation and insulin-stimulated glucose uptake. J Biol Chem 2009, 284:10755-10763.

62. Das RM, Van Hateren NJ, Howell GR, Farrell ER, Bangs FK, Porteous VC, Manning EM, McGrew MJ, Ohyama K, Sacco MA, Halley PA, Sang HM, Storey KG: A robust system for RNA interference in the chicken using a modified microRNA operon. Dev Biol 2006, 294:554-563.

63. Wakamatsu Y, Maynard TM, Jones SU, Weston JA: NUMB localizes in the basal cortex of mitotic avian neuroepithelial cells and modulates neuronal differentiation by binding to NOTCH-1. Neuron 1999, 23:71-81.

64. Kohyama J, Tokunaga A, Fujita Y, Miyoshi H, Nagai T, Miyawaki A, Nakao K, Matsuzaki Y, Okano H: Visualization of spatiotemporal activation of Notch signaling: live monitoring and significance in neural development. Dev Biol 2005, 286:311-325.

65. Gerhardt $\mathrm{H}$, Betsholtz C: Endothelial-pericyte interactions in angiogenesis. Cell Tissue Res 2003, 314:15-23.

doi:10.1186/s12915-014-0053-9

Cite this article as: Applebaum et al.: Segregation of striated and smooth muscle lineages by a Notch-dependent regulatory network. BMC Biology 2014 12:53.

\section{Submit your next manuscript to BioMed Central and take full advantage of:}

- Convenient online submission

- Thorough peer review

- No space constraints or color figure charges

- Immediate publication on acceptance

- Inclusion in PubMed, CAS, Scopus and Google Scholar

- Research which is freely available for redistribution 